\title{
Exploding solutions for a nonlocal quadratic evolution problem
}

\section{Dong Li, José L. Rodrigo and Xiaoyi Zhang}

\begin{abstract}
We consider a nonlinear parabolic equation with fractional diffusion which arises from modelling chemotaxis in bacteria. We prove the wellposedness, continuation criteria and smoothness of local solutions. In the repulsive case we prove global wellposedness in Sobolev spaces. Finally in the attractive case, we prove that for a class of smooth initial data the $L_{x}^{\infty}$-norm of the corresponding solution blows up in finite time. This solves a problem left open by Biler and Woyczyński [8].
\end{abstract}

\section{Introduction and main results}

In this paper we consider the following evolution equation on $\mathbb{R}^{2}$ :

$$
\left\{\begin{array}{l}
\partial_{t} u=-\nu(-\Delta)^{\alpha / 2} u-\nabla \cdot(u B(u)), \quad 0<\alpha<2, \\
u(0, x)=u_{0}(x),
\end{array}\right.
$$

where $\nu \geq 0$ is the viscosity coefficient. Physically meaningful solutions of (1.1) are nonnegative functions $u: \mathbb{R}^{2} \times \mathbb{R}^{+} \rightarrow \mathbb{R}$ which represent the density of particles in $\mathbb{R}^{2}$. The term $B(u)$ is a linear integral operator given by

$$
B(u)(x)=\mu \int_{\mathbb{R}^{2}} \frac{x-y}{|x-y|^{2}} u(y) d y,
$$

and $\mu= \pm 1$. When $\mu=-1$ we will call $B$ an attractive kernel, while the case $\mu=1$ corresponds to a repulsive kernel. By explicit computation it is easy to see that $B(u)$ has an equivalent expression:

$$
B(u)=-C_{1} \mu(-\Delta)^{-1} \nabla u,
$$

where $C_{1}>0$ is an absolute constant.

2000 Mathematics Subject Classification: 35A05, 35A07, 35K55, 35K57.

Keywords: Nonlinear parabolic equation, fractional diffusion, chemotaxis. 
Throughout the paper, we shall use both expressions of $B(u)$ whenever it is convenient. The fractional Laplacian $(-\Delta)^{\alpha / 2}$ is defined via the Fourier transform:

$$
\left(\widehat{-\Delta)^{\alpha / 2}} u(\xi)=|\xi|^{\alpha} \hat{u}(\xi)\right.
$$

where $\hat{u}$ denotes the Fourier transform of $u$.

Equation (1.1) describes many physical processes involving diffusion and interaction of particles (see for example [9] and [8]). When $\alpha=2$, the attractive case $\mu=-1$ models the evolution of particles in a cloud interacting via gravitational attraction (see [23] for more details). In this case (1.1) can also be regarded as a simplification of the classical Keller-Segel model [14]. On the other hand, the repulsive case $\mu=1$ models the Brownian diffusion of charged particles with Coulomb repulsion (see [2]). The regime $0<\alpha<2$ was studied in [8] and it corresponds to the so-called anomalous diffusion which in probabilistic terms has a connection with stochastic equations driven by Lévy $\alpha$-stable processes. As mentioned in [8], an important technical difficulty lies in the fact that non-Gaussian Lévy $\alpha$-stable $(0<\alpha<2)$ semigroups have densities which decay only at an algebraic rate $|x|^{-2-\alpha}$ as $|x| \rightarrow \infty$ while the Gaussian kernel $\alpha=2$ decays exponentially fast. Equation (1.1) shares a similar form with several other models with different choices of the kernel $B$. For example, if one takes $B(u)=(-\Delta)^{-1} \nabla^{\perp} u$, where $\nabla^{\perp} u:=\left(-\partial_{x_{2}} u, \partial_{x_{1}} u\right)$, then equation (1.1) with this choice of $B$ and $\nu=0$ is the $2 \mathrm{D}$ Euler equation in vorticity form for which global solutions exist under rather general conditions on the initial data (see for example [20]). Another closely related model is the surface quasi-geostrophic equation for which $B(u)=(-\Delta)^{-1 / 2} \nabla^{\perp} u([10])$. We refer the interested reader to $[1,12,15,16,17,18,19,21]$ where other equations with fractal type diffusion have been considered. We also mention that analogous problems of (1.1) in bounded domains were studied in $[2,3,4,5,6,7]$.

The main goal of this paper is to study in detail classical solutions of (1.1) in the unbounded domain $\mathbb{R}^{2}$. Depending on the sign of the interaction kernel $B$, we investigate conditions for global in time existence or finite time blowup. Part of the results obtained here complement those of [8], where blowup in the case $\alpha=2$ and $\mu=-1$ was obtained. In particular we solve a problem left open by Biler and Woyczyński [8], namely the existence of blowing-up solutions in the case $\mu=-1$ and $0<\alpha<2$ (see, for example, [8, Proposition 4.1] and preceding remarks there; see also Theorem 1.10 below). In the case $\alpha=2$ and $\mu=-1$ considered in [8], the existence of blowing-up solutions is proved by a virial argument. More precisely one studies the evolution of the integral $\int u|x|^{2} d x$ and proves that the ODE associated to its evolution generates a negative solution in finite time for sufficiently large initial data. This argument no longer works in the fractal case $0<\alpha<2$ 
since the weight function $|x|^{2}$ makes the linear term too strong to be controlled by the nonlinear part. To solve this problem, we use a truncated virial argument choosing the same weight function but multiplied by a smooth cutoff function. We then control the remainders by proving a mass localization lemma (see Lemma 7.4) which prevents the mass from evacuation for a short time. For a properly chosen time interval and sufficiently localized initial data, we show that the truncated virial expression which is nonnegative becomes negative in finite time and thus obtain a contradiction.

We now state more precisely our main results. The first theorem establishes the local wellposedness of (1.1) in Sobolev space.

Theorem 1.1. (Local existence and uniqueness of solutions). Let $\mu= \pm 1$ and $\nu \geq 0$. For any $u_{0} \in H_{x}^{s}\left(\mathbb{R}^{2}\right) \cap L_{x}^{q}\left(\mathbb{R}^{2}\right)$ with $s>3$ and $1<q<2$, there exists $T=T\left(\left\|u_{0}\right\|_{H_{x}^{2}\left(\mathbb{R}^{2}\right)},\left\|u_{0}\right\|_{L_{x}^{q}\left(\mathbb{R}^{2}\right)}\right)>0$ and a unique solution $u$ of (1.1) in $C\left([0, T), H_{x}^{s}\left(\mathbb{R}^{2}\right) \cap L_{x}^{q}\left(\mathbb{R}^{2}\right)\right)$. Moreover we have $u \in C^{1}\left([0, T), H_{x}^{s_{0}}\left(\mathbb{R}^{2}\right)\right)$, where $s_{0}=\min \{s-\alpha, s-1\}>1$.

Remark 1.2. In the local theory we do not assume that $u_{0}$ is nonnegative. The assumption $s>3$ in Theorem 1.1 can be weakened further although we shall not do it here. We chose these conditions simply for the sake of convenience. If $\nu=0$, the constructed solution is in fact in $C^{1}\left([0, T), H_{x}^{s-1}\left(\mathbb{R}^{2}\right)\right)$.

Remark 1.3. The analysis here should be compared with the case $\alpha=2$ in [8]. There (see [8, Remark 3.2]) it is mentioned that a small data result can be proved by using a perturbation argument. A conditional result for classical solutions is also proved there for bounded domains with no-flux boundary condition (see [8, Section 6]).

The next two results establish the continuation criteria of solutions and further properties of the solutions.

Theorem 1.4. (Blow up -continuation of solutions criteria). Let $\mu= \pm 1$ and $\nu \geq 0$. Let $u_{0} \in H_{x}^{s}\left(\mathbb{R}^{2}\right) \cap L_{x}^{q}\left(\mathbb{R}^{2}\right)$ with $s>3$ and $1<q<2$. Assume $u \in C\left([0, T), H_{x}^{s}\left(\mathbb{R}^{2}\right) \cap L_{x}^{q}\left(\mathbb{R}^{2}\right)\right)$ is the maximal-lifespan solution obtained in Theorem 1.1. Then either $T=+\infty$, in which case we have a global solution, or $T<\infty$, and we have

$$
\lim _{t \rightarrow T} \int_{0}^{t}\|u(s)\|_{L_{x}^{\infty}\left(\mathbb{R}^{2}\right)} d s=+\infty .
$$

Theorem 1.5. (Further properties of solutions). Assume $\mu= \pm 1$ and $\nu \geq 0$. Let $u_{0} \in H_{x}^{s}\left(\mathbb{R}^{2}\right) \cap L_{x}^{q}\left(\mathbb{R}^{2}\right)$ with $s>3$ and $1<q<2$. If $u_{0} \geq 0$, then the corresponding solution obtained in Theorem 1.1 remains non-negative, i.e. $u(t) \geq 0$ for any $0 \leq t<T$. If in addition $u_{0} \in L_{x}^{1}\left(\mathbb{R}^{2}\right)$, then $\|u(x, t)\|_{L_{x}^{1}\left(\mathbb{R}^{2}\right)}=\left\|u_{0}\right\|_{L_{x}^{1}\left(\mathbb{R}^{2}\right)}$ for any $0 \leq t<T$. 
Remark 1.6. If $u_{0} \in H_{x}^{s}\left(\mathbb{R}^{2}\right) \cap L_{x}^{1}\left(\mathbb{R}^{2}\right)$ for some $s>3$ and $u_{0} \geq 0$, then by an elementary argument $u_{0} \in H_{x}^{s}\left(\mathbb{R}^{2}\right) \cap L_{x}^{q}\left(\mathbb{R}^{2}\right)$ for any $1<q<\infty$. Theorem 1.5 asserts that we can obtain a solution in $C\left([0, T), H_{x}^{s}\left(\mathbb{R}^{2}\right) \cap L_{x}^{q}\left(\mathbb{R}^{2}\right)\right)$ in any $1<q<2$. Note that although we prove that the $L_{x}^{1}$ norm of $u$ is preserved, the additional strong continuity in $C\left([0, T), L_{x}^{1}\right)$ is not shown here. We conjecture this is the case.

The following theorem deals with the case $\mu=1$, proving that the solution to (1.1) is global.

Theorem 1.7. (Global well-posedness in the repulsive case). Assume $\mu=1$ and $\nu \geq 0$. Let $u_{0} \in H_{x}^{s}\left(\mathbb{R}^{2}\right) \cap L_{x}^{q}\left(\mathbb{R}^{2}\right)$ with $s>3,1 \leq q<2$ and $u_{0} \geq 0$. Then the corresponding solution obtained as in Theorem 1.1 is global. More precisely, if $1<q<2$, then $u \in C\left([0, \infty), H_{x}^{s}\left(\mathbb{R}^{2}\right) \cap L_{x}^{q}\left(\mathbb{R}^{2}\right)\right)$. If $q=1$, then $u \in C\left([0, \infty), H_{x}^{s}\left(\mathbb{R}^{2}\right) \cap L_{x}^{r}\left(\mathbb{R}^{2}\right)\right)$ for any $1<r<2$ and $\|u(t)\|_{L_{x}^{1}\left(\mathbb{R}^{2}\right)}=$ $\left\|u_{0}\right\|_{L_{x}^{1}\left(\mathbb{R}^{2}\right)}$ for any $t \geq 0$. In all cases $(\nu \geq 0$ ) and for all $1 \leq r \leq \infty$, the $L_{x}^{r}$-norm of $u$ is non-increasing, i.e., for any $0 \leq t_{1}<t_{2}<\infty$, we have

$$
\left\|u\left(t_{1}\right)\right\|_{L_{x}^{r}\left(\mathbb{R}^{2}\right)} \geq\left\|u\left(t_{2}\right)\right\|_{L_{x}^{r}\left(\mathbb{R}^{2}\right)} .
$$

In the inviscid case $(\nu=0)$, with $q=1$ we have more precise estimates. They are as follows. For any $1 \leq p \leq \infty$, we have

$$
\begin{aligned}
& \left\|u_{0}\right\|_{L_{x}^{p}\left(\mathbb{R}^{2}\right)} \cdot\left(1+C_{1}\left\|u_{0}\right\|_{L_{x}^{\infty}\left(\mathbb{R}^{2}\right)} t\right)^{-\left(1-\frac{1}{p}\right)} \leq\|u(t)\|_{L_{x}^{p}\left(\mathbb{R}^{2}\right)} \leq \\
& \quad \leq\left\|u_{0}\right\|_{L_{x}^{1}\left(\mathbb{R}^{2}\right)}^{\frac{1}{p}} \cdot\left\|u_{0}\right\|_{L_{x}^{\infty}\left(\mathbb{R}^{2}\right)}^{1-\frac{1}{p}} \cdot\left(1+C_{1}\left\|u_{0}\right\|_{L_{x}^{\infty}\left(\mathbb{R}^{2}\right)} t\right)^{-\left(1-\frac{1}{p}\right)}, \quad \forall t \geq 0,
\end{aligned}
$$

where $C_{1}$ is the same constant as in (1.3).

Remark 1.8. Note that the estimate (1.4) shows that in the inviscid case $(\nu=0)$, the $L_{x}^{p}$-norm of $u$ decays only algebraically fast. In particular for $1<p \leq \infty$ the estimate is sharp and the $L_{x}^{p}$-norm of $u$ decays at a rate exactly proportional to $t^{-\left(1-\frac{1}{p}\right)}$ (provided, of course, that the initial data $u_{0}$ does not vanish identically). More precisely we have the following theorem.

In the case with no diffusion and $\mu=-1$, we expect the blowup of solutions for generic initial data (even for initial data that are not necessarily nonnegative).

Theorem 1.9. (Blowup in the attractive case with no diffusion). Assume $\mu=-1$ and $\nu=0$. Let $u_{0} \in H_{x}^{s}\left(\mathbb{R}^{2}\right) \cap L_{x}^{q}\left(\mathbb{R}^{2}\right)$ for some $1<q<2, s>3\left(u_{0}\right.$ is not necessarily nonnegative) and $u$ be the corresponding maximal-lifespan solution. Assume the set $\left\{y \in \mathbb{R}^{2}: u_{0}(y)>0\right\}$ is nonempty and define the blowup time $T$ by

$$
T:=\inf _{y \in \mathbb{R}^{2}, u_{0}(y)>0} \frac{1}{C_{1} u_{0}(y)},
$$

where $C_{1}$ is the same constant as in (1.3). 
Then the maximal lifespan of $u$ is exactly given by $[0, T)$. More precisely, we have $u \in C\left([0, T), L_{x}^{q}\left(\mathbb{R}^{2}\right) \cap H_{x}^{s}\left(\mathbb{R}^{2}\right)\right)$ and the following sharp estimate holds:

$$
\frac{1}{C_{1} \cdot(T-t)} \leq\|u(t)\|_{L_{x}^{\infty}\left(\mathbb{R}^{2}\right)} \leq \max \left\{\left\|u_{0}\right\|_{L_{x}^{\infty}\left(\mathbb{R}^{2}\right)}, \frac{1}{C_{1} \cdot(T-t)}\right\},
$$

for all $t<T$. In particular this implies that $\lim _{t \rightarrow T}\|u(t)\|_{L_{x}^{\infty}\left(\mathbb{R}^{2}\right)}=\infty$.

The last result gives the existence of blowing-up solutions for a class of well-chosen initial data. Here $A_{\delta, a, b}$ denotes a class of functions defined precisely below (Definition 7.1).

Theorem 1.10. (Finite time blow up in the attractive, diffusive case). Assume $\mu=-1$ and $\nu>0$. Let $u_{0} \in H_{x}^{s}\left(\mathbb{R}^{2}\right) \cap L_{x}^{1}\left(\mathbb{R}^{2}\right)$ with $s>3$ and $u_{0} \geq 0$. Then there exists an open set of parameters $\delta, a, b$ such that if $u_{0} \in A_{\delta, a, b}$, then the corresponding solution of equation (1.1) blows up in finite time. More precisely, there exists $T<\infty$, such that

$$
\lim _{t \rightarrow T}\|u(t)\|_{L_{x}^{\infty}\left(\mathbb{R}^{2}\right)}=+\infty .
$$

Outline of the paper. This paper is organized as follows. In Section 2 we collect some basic estimates and preparatory lemmas. Section 3 is devoted to the proof of local wellposedness in Sobolev spaces (Theorem 1.1). The proofs of Theorem 1.4, 1.5, 1.7 and 1.9 are given in Section 4, Section 5 and Section 6. Lastly in Section 7 we prove the existence of blowing-up solutions (Theorem 1.10).

\section{Preliminaries}

In this section we compile the notation, auxiliary results from harmonic analysis and other lemmas used throughout the paper.

Notation. Throughout the paper we denote by $L_{x}^{p}=L_{x}^{p}\left(\mathbb{R}^{2}\right)$, for $1 \leq p \leq \infty$, the usual Lebesgue spaces on $\mathbb{R}^{2}$. For $s>0, s$ being an integer and $1 \leq p \leq \infty, W_{x}^{s, p}=W_{x}^{s, p}\left(\mathbb{R}^{2}\right)$ denotes the usual Sobolev space

$$
W_{x}^{s, p}=\left\{f \in S^{\prime}\left(\mathbb{R}^{2}\right):\|f\|_{W^{s, p}}=\sum_{0 \leq|j| \leq s}\left\|\partial_{x}^{j} f\right\|_{L_{x}^{p}\left(\mathbb{R}^{2}\right)}<\infty\right\} .
$$

When $p=2$, we write $H_{x}^{s}=H_{x}^{s}\left(\mathbb{R}^{2}\right)=W_{x}^{s, 2}\left(\mathbb{R}^{2}\right)$ and $\|\cdot\|_{H_{x}^{m}}$ as its norm. We will also use the Sobolev space of fractional power $H_{x}^{s}\left(\mathbb{R}^{2}\right), s \in \mathbb{R}$, which is defined via the Fourier transform:

$$
\|f\|_{H^{s}}=\left\|(1+|\xi|)^{s} \hat{f}(\xi)\right\|_{L_{\xi}^{2}} .
$$


For any $s \geq 0$, the space $C_{W}\left([0, T) ; H_{x}^{s}\left(\mathbb{R}^{2}\right)\right)$ consists of functions which are continuous in the weak topology of $H_{x}^{s}$, i.e., $u \in C_{W}\left([0, T) ; H_{x}^{s}\left(\mathbb{R}^{2}\right)\right)$ if and only if for any $\phi \in H_{x}^{s}\left(\mathbb{R}^{2}\right)$, the scalar product $(\phi, u(t))_{s}$ is a continuous function of $t$ on $[0, T)$, where

$$
(\phi, u)_{s}=\int_{\mathbb{R}^{2}} \hat{\phi}(\xi) \hat{u}(\xi)(1+|\xi|)^{2 s} d \xi .
$$

Finally, for any two quantities $X$ and $Y$, we use $X \lesssim Y$ or $Y \gtrsim X$ whenever $X \leq C Y$ for some constant $C>0$. A constant $C$ with subscripts implies the dependence on these parameters. We use $X \sim Y$ if both $X \lesssim Y$ and $Y \lesssim X$ holds.

\subsection{Basic harmonic analysis}

Let $\varphi(\xi)$ be a radial bump function supported in the ball $\left\{\xi \in \mathbb{R}^{d}:|\xi| \leq \frac{11}{10}\right\}$ and equal to 1 on the ball $\left\{\xi \in \mathbb{R}^{d}:|\xi| \leq 1\right\}$. For each number $N \in \mathbb{Z}$, we define the Fourier multipliers

$$
\begin{aligned}
\widehat{P_{\leq N} f}(\xi) & :=\varphi\left(2^{-N} \xi\right) \hat{f}(\xi) \\
\widehat{P_{>N} f}(\xi) & :=\left(1-\varphi\left(2^{-N} \xi\right)\right) \hat{f}(\xi) \\
\widehat{P_{N} f}(\xi) & :=\psi\left(2^{-N} \xi\right) \hat{f}(\xi):=\left(\varphi\left(2^{-N} \xi\right)-\varphi\left(2^{-N+1} \xi\right)\right) \hat{f}(\xi)
\end{aligned}
$$

and similarly $P_{<N}$ and $P_{\geq N}$. We also define

$$
P_{M<\cdot \leq N}:=P_{\leq N}-P_{\leq M}=\sum_{M<N^{\prime} \leq N} P_{N^{\prime}}
$$

whenever $M<N$.

Lemma 2.2. (Bernstein estimates). For $1 \leq p \leq q \leq \infty$,

$$
\begin{aligned}
\left\||\nabla|^{ \pm s} P_{N} f\right\|_{L_{x}^{p}\left(\mathbb{R}^{2}\right)} & \sim 2^{ \pm s N}\left\|P_{N} f\right\|_{L_{x}^{p}\left(\mathbb{R}^{2}\right)}, \\
\left\|P_{\leq N} f\right\|_{L_{x}^{q}\left(\mathbb{R}^{2}\right)} & \lesssim 2^{\left(\frac{2}{p}-\frac{2}{q}\right) N}\left\|P_{\leq N} f\right\|_{L_{x}^{p}\left(\mathbb{R}^{2}\right)}, \\
\left\|P_{N} f\right\|_{L_{x}^{q}\left(\mathbb{R}^{2}\right)} & \lesssim 2^{\left(\frac{2}{p}-\frac{2}{q}\right) N}\left\|P_{N} f\right\|_{L_{x}^{p}\left(\mathbb{R}^{2}\right)}
\end{aligned}
$$

Lemma 2.3. (Commutator estimate). For any $f, g \in \mathcal{S}\left(\mathbb{R}^{2}\right)$, consider the commutator

$$
\left[P_{k} D, f\right] g=P_{k} D(f g)-f P_{k} D g .
$$

We have, for any $1 \leq p \leq \infty$,

$$
\left\|\left[P_{k} D, f\right] g\right\|_{L_{x}^{p}\left(\mathbb{R}^{2}\right)} \lesssim\|D f\|_{L_{x}^{\infty}\left(\mathbb{R}^{2}\right)}\|g\|_{L_{x}^{p}\left(\mathbb{R}^{2}\right)} .
$$


Proof. We have

$$
\begin{aligned}
\mid\left(P_{k} D(f g)\right)(x)- & f(x)\left(P_{k} D g\right)(x) \mid= \\
= & \left|\int_{\mathbb{R}^{2}} 2^{3 k}(D \hat{\psi})\left(2^{k}(x-y)\right) g(y)(f(x)-f(y)) d y\right| \\
\leq & \|D f\|_{L_{x}^{\infty}\left(\mathbb{R}^{2}\right)} \int_{\mathbb{R}^{2}} 2^{2 k} 2^{k}|x-y|\left|(D \hat{\psi})\left(2^{k}(x-y)\right)\right||g(y)| d y
\end{aligned}
$$

Define $\psi_{1}(x)=|x|(D \hat{\psi})(x)$, then by Minkowski's inequality we have $\left\|\left[P_{k} D, f\right] g\right\|_{L_{x}^{p}\left(\mathbb{R}^{2}\right)} \lesssim\|D f\|_{L_{x}^{\infty}\left(\mathbb{R}^{2}\right)}\left\|\psi_{1}\right\|_{L_{x}^{1}\left(\mathbb{R}^{2}\right)}\|g\|_{L_{x}^{p}\left(\mathbb{R}^{2}\right)} \lesssim\|D f\|_{L_{x}^{\infty}\left(\mathbb{R}^{2}\right)}\|g\|_{L_{x}^{p}\left(\mathbb{R}^{2}\right)}$.

Lemma 2.4. Let $s \geq 0$, then

$$
\begin{aligned}
& \sum_{k>0} 2^{2 k s}\left|\int_{\mathbb{R}^{2}} P_{k} D(u f) P_{k} u d x\right| \lesssim \\
& \quad \lesssim\left(\|u\|_{L_{x}^{\infty}\left(\mathbb{R}^{2}\right)}+\|D f\|_{L_{x}^{\infty}\left(\mathbb{R}^{2}\right)}\right)\left\|P_{>-20} u\right\|_{\dot{H}_{x}^{s}\left(\mathbb{R}^{2}\right)}^{2}+\|u\|_{L_{x}^{\infty}\left(\mathbb{R}^{2}\right)}\left\|P_{>-20} f\right\|_{\dot{H}_{x}^{s+1}\left(\mathbb{R}^{2}\right)}^{2} .
\end{aligned}
$$

Proof. By frequency localization, we have

$$
\begin{aligned}
& \int_{\mathbb{R}^{2}} P_{k} D(u f) P_{k} u d x= \\
&=\int_{\mathbb{R}^{2}} P_{k} D\left(u f_{\leq k-5}\right) P_{k} u d x+\int_{\mathbb{R}^{2}} P_{k} D\left(u f_{\geq k-4}\right) P_{k} u d x \\
&=\int_{\mathbb{R}^{2}}\left[P_{k} D, f_{\leq k-5}\right] u P_{k} u d x+\int_{\mathbb{R}^{2}} f_{\leq k-5}\left(P_{k} D u\right) P_{k} u d x \\
& \quad+\int_{\mathbb{R}^{2}} P_{k} D\left(u f_{\geq k-4}\right) P_{k} u d x \\
&=\int_{\mathbb{R}^{2}}\left[P_{k} D, f_{\leq k-5}\right] u_{[k-9, k+9]} P_{k} u d x-\frac{1}{2} \int_{\mathbb{R}^{2}}\left(D f_{\leq k-5}\right)\left|P_{k} u\right|^{2} d x \\
& \quad+\int_{\mathbb{R}^{2}} P_{k} D\left(u f_{[k-4, k+8]}\right) P_{k} u d x+\int_{\mathbb{R}^{2}} P_{k} D\left(u f_{\geq k+9}\right) P_{k} u d x \\
&=: A_{k}+B_{k}+C_{k}+D_{k} .
\end{aligned}
$$

The assumption $s \geq 0$ will be needed in the estimate of $D_{k}$. We begin with

Estimate of $A_{k}$. By Lemma 2.3, we have

$$
\left|A_{k}\right| \lesssim\|D f\|_{L_{x}^{\infty}\left(\mathbb{R}^{2}\right)}\left\|u_{[k-9, k+9]}\right\|_{L_{x}^{2}\left(\mathbb{R}^{2}\right)}\left\|P_{k} u\right\|_{L_{x}^{2}\left(\mathbb{R}^{2}\right)}
$$


Clearly then

$$
\begin{aligned}
\sum_{k>0} 2^{2 k s}\left|A_{k}\right| & \lesssim\|D f\|_{L_{x}^{\infty}\left(\mathbb{R}^{2}\right)} \sum_{k>0} 2^{k s}\left(\left\|u_{[k-9, k+9]}\right\|_{L_{x}^{2}\left(\mathbb{R}^{2}\right)}^{2}+\left\|P_{k} u\right\|_{L_{x}^{2}\left(\mathbb{R}^{2}\right)}^{2}\right) \\
& \lesssim\|D f\|_{L_{x}^{\infty}\left(\mathbb{R}^{2}\right)} \sum_{k>-10} 2^{2 k s}\left\|P_{k} u\right\|_{L_{x}^{2}\left(\mathbb{R}^{2}\right)}^{2} .
\end{aligned}
$$

This will be enough for us.

Estimate of $B_{k}$. This is rather straightforward. We have

$$
\sum_{k>0} 2^{2 k s}\left|B_{k}\right| \lesssim\|D f\|_{L_{x}^{\infty}\left(\mathbb{R}^{2}\right)} \sum_{k>0} 2^{2 k s}\left\|P_{k} u\right\|_{L_{x}^{2}\left(\mathbb{R}^{2}\right)}^{2},
$$

which will suffice to complete the proof.

Estimate of $C_{k}$. By integration by parts and Bernstein's inequality, we have

$$
\begin{aligned}
\sum_{k>0} 2^{2 k s}\left|C_{k}\right| & =\sum_{k>0} 2^{2 k s}\left|\int_{\mathbb{R}^{2}} u f_{[k-4, k+8]} P_{k}^{2} D u d x\right| \\
& \lesssim \sum_{k>0} 2^{2 k s}\|u\|_{L_{x}^{\infty}\left(\mathbb{R}^{2}\right)}\left\|f_{[k-4, k+8]}\right\|_{L_{x}^{2}\left(\mathbb{R}^{2}\right)} 2^{k}\left\|P_{k} u\right\|_{L_{x}^{2}\left(\mathbb{R}^{2}\right)} \\
& \lesssim\|u\|_{L_{x}^{\infty}\left(\mathbb{R}^{2}\right)} \sum_{k>0} 2^{2 k s}\left(\left(2^{k}\left\|f_{[k-4, k+8]}\right\|_{L_{x}^{2}\left(\mathbb{R}^{2}\right)}\right)^{2}+\left\|P_{k} u\right\|_{L_{x}^{2}\left(\mathbb{R}^{2}\right)}^{2}\right) \\
& \lesssim\|u\|_{L_{x}^{\infty}\left(\mathbb{R}^{2}\right)} \sum_{k>-10}\left(2^{2 k(s+1)}\left\|P_{k} f\right\|_{L_{x}^{2}\left(\mathbb{R}^{2}\right)}^{2}+2^{2 k s}\left\|P_{k} u\right\|_{L_{x}^{2}\left(\mathbb{R}^{2}\right)}^{2}\right)
\end{aligned}
$$

This is again sufficient.

Estimate of $D_{k}$. By frequency localization, we have

$$
\begin{aligned}
D_{k} & =\int_{\mathbb{R}^{2}} P_{k} D\left(u_{\geq k+3} f_{\geq k+9}\right) P_{k} u d x \\
& =\sum_{\substack{j \geq k+9, j^{\prime} \geq k+3 \\
\left|j-j^{\prime}\right| \leq 1}} \int_{\mathbb{R}^{2}} P_{k} D\left(u_{j^{\prime}} f_{j}\right) P_{k} u d x=\sum_{\substack{j \geq k+9, j^{\prime} \geq k+3 \\
\left|j-j^{\prime}\right| \leq 1}} \int_{\mathbb{R}^{2}} u_{j^{\prime}} f_{j} P_{k}^{2} D u d x .
\end{aligned}
$$

By Bernstein's inequality, we then have

$$
\left|D_{k}\right| \lesssim \sum_{\substack{j \geq k+9, j^{\prime} \geq k+3 \\\left|j-j^{\prime}\right| \leq 1}}\left\|u_{j^{\prime}}\right\|_{L_{x}^{2}\left(\mathbb{R}^{2}\right)}\left\|f_{j}\right\|_{L_{x}^{2}\left(\mathbb{R}^{2}\right)} 2^{k}\|u\|_{L_{x}^{\infty}\left(\mathbb{R}^{2}\right)}
$$


Therefore, interchanging the sums over $j$ and $k$ (here we use the fact $s \geq 0$ to get $\left.\sum_{0<k<j} 2^{k(2 s+1)} \lesssim 2^{j(2 s+1)}\right)$, we obtain

$$
\begin{aligned}
\sum_{k>0} 2^{k s}\left|D_{k}\right| & \lesssim \sum_{k>0} 2^{2 k s} \sum_{\substack{j \geq k+9, j^{\prime} \geq k+3 \\
\left|j-j^{\prime}\right| \leq 1}} 2^{k}\left\|u_{j^{\prime}}\right\|_{L_{x}^{2}\left(\mathbb{R}^{2}\right)}\left\|f_{j}\right\|_{L_{x}^{2}\left(\mathbb{R}^{2}\right)}\|u\|_{L_{x}^{\infty}\left(\mathbb{R}^{2}\right)} \\
& \lesssim \sum_{\substack{j \geq 9, j^{\prime} \geq 3 \\
\left|j-j^{\prime}\right| \leq 1}} 2^{2 j s} \cdot 2^{j}\left\|u_{j^{\prime}}\right\|_{L_{x}^{2}\left(\mathbb{R}^{2}\right)}\left\|f_{j}\right\|_{L_{x}^{2}\left(\mathbb{R}^{2}\right)}\|u\|_{L_{x}^{\infty}\left(\mathbb{R}^{2}\right)} \\
& \lesssim\|u\|_{L_{x}^{\infty}\left(\mathbb{R}^{2}\right)} \sum_{j>0}\left(2^{2 j(s+1)}\left\|f_{j}\right\|_{L_{x}^{2}\left(\mathbb{R}^{2}\right)}^{2}+2^{2 j s}\left\|u_{j}\right\|_{L_{x}^{2}\left(\mathbb{R}^{2}\right)}^{2}\right) .
\end{aligned}
$$

This is clearly good for us. The lemma is proved.

Lemma 2.5. Given $u \in H^{s}\left(\mathbb{R}^{2}\right) \cap L^{q}\left(\mathbb{R}^{2}\right)$, for $s>1$ and $1 \leq q \leq 2$, we have

$$
\begin{aligned}
\|u\|_{L_{x}^{\infty}\left(\mathbb{R}^{2}\right)}+\|D B(u)\|_{L_{x}^{\infty}\left(\mathbb{R}^{2}\right)}+\|u\|_{\dot{H}_{x}^{s}\left(\mathbb{R}^{2}\right)} & \lesssim \\
& \lesssim\|u\|_{L_{x}^{q}\left(\mathbb{R}^{2}\right)}+\left(\sum_{k>0} 2^{2 k s}\left\|P_{k} u\right\|_{L_{x}^{2}\left(\mathbb{R}^{2}\right)}^{2}\right)^{\frac{1}{2}} .
\end{aligned}
$$

with $B$ as in (1.2).

Proof. The proof is elementary. We only sketch the main ideas. For $k \leq 0$, by Bernstein's inequality we have (using that $q \leq 2$ )

$$
\left\|P_{k} u\right\|_{L_{x}^{\infty}\left(\mathbb{R}^{2}\right)}+\left\|P_{k} D B(u)\right\|_{L_{x}^{\infty}\left(\mathbb{R}^{2}\right)}+\left\|P_{k} u\right\|_{\dot{H}_{x}^{s}\left(\mathbb{R}^{2}\right)} \lesssim\left(2^{k}+2^{k s}\right)\|u\|_{L_{x}^{q}\left(\mathbb{R}^{2}\right)} .
$$

For $k \geq 0$, we also have

$$
\left\|P_{k} u\right\|_{L_{x}^{\infty}\left(\mathbb{R}^{2}\right)}+\left\|P_{k} D B(u)\right\|_{L_{x}^{\infty}\left(\mathbb{R}^{2}\right)} \lesssim 2^{k(1-s)} \cdot 2^{k s}\left\|P_{k} u\right\|_{L_{x}^{2}\left(\mathbb{R}^{2}\right)} .
$$

Note that the high frequency part of the $\dot{H}_{x}^{s}$ norm is already bounded by the RHS of (2.2). Summing over all $k \in \mathbb{Z}$ and applying Cauchy-Schwartz quickly yields the result.

Lemma 2.6. Given $u \in \dot{H}^{2}\left(\mathbb{R}^{2}\right) \cap L^{\infty}\left(\mathbb{R}^{2}\right)$, let Bu be as in (1.2). We have

$$
\|D B(u)\|_{L_{x}^{\infty}\left(\mathbb{R}^{2}\right)} \lesssim \log \left(e+\|u\|_{\dot{H}_{x}^{2}\left(\mathbb{R}^{2}\right)}\right)\left(1+\|u\|_{L_{x}^{\infty}\left(\mathbb{R}^{2}\right)}\right)+\|u\|_{L_{x}^{2}\left(\mathbb{R}^{2}\right)} .
$$

Proof. Let $k_{0}$ be a number to be chosen later. By Bernstein's inequality we have

$$
\begin{aligned}
\|D B(u)\|_{L_{x}^{\infty}\left(\mathbb{R}^{2}\right)} \lesssim & \sum_{k>k_{0}} 2^{-k}\left\|P_{k} u\right\|_{\dot{H}_{x}^{2}\left(\mathbb{R}^{2}\right)}+\sum_{k \leq 0} 2^{k}\left\|P_{k} u\right\|_{L_{x}^{2}\left(\mathbb{R}^{2}\right)} \\
& \quad+\sum_{0<k<k_{0}}\left\|P_{k} u\right\|_{L_{x}^{\infty}\left(\mathbb{R}^{2}\right)} \\
\lesssim & 2^{-k_{0}}\|u\|_{\dot{H}_{x}^{2}\left(\mathbb{R}^{2}\right)}+\|u\|_{L_{x}^{2}\left(\mathbb{R}^{2}\right)}+k_{0}\|u\|_{L_{x}^{\infty}\left(\mathbb{R}^{2}\right)}
\end{aligned}
$$


If $\|u\|_{\dot{H}_{x}^{2}\left(\mathbb{R}^{2}\right)} \leq 2 e$ then just choose $k_{0}=1$.

Otherwise choose $k_{0}=\left[\log _{2}\left(\|u\|_{\dot{H}_{x}^{2}\left(\mathbb{R}^{2}\right)}\right)\right]$ (here $[x]$ denotes the integer part of $x$ ) and this quickly yields the result.

Lemma 2.7. (Positivity lemma). Let $0<\alpha \leq 2, T>0$. Denote $\Omega_{T}=$ $(0, T] \times \mathbb{R}^{2}$. Let $u \in C_{t, x}^{1,2}\left(\Omega_{T}\right) \cap C_{t, x}^{0}\left(\bar{\Omega}_{T}\right) \cap L_{t, x}^{p}\left(\Omega_{T}\right)$ for some $1 \leq p<\infty$. Assume $g: \mathbb{R}^{2} \rightarrow \mathbb{R}, g \in C\left(\mathbb{R}^{2}\right), f: \Omega_{T} \rightarrow \mathbb{R}^{2}$ and $f \in C_{t, x}^{0,1}\left(\Omega_{T}\right)$ are given functions and the following conditions hold.

1. $u$ satisfies the following inequality pointwise:

$$
\left\{\begin{array}{l}
\partial_{t} u+\nabla \cdot(f u) \geq-\nu(-\Delta)^{\alpha / 2} u, \quad(t, x) \in \Omega_{T}, \\
u(0, x)=g(x), \quad x \in \mathbb{R}^{2} .
\end{array}\right.
$$

Here $\nu \geq 0$ is the viscosity coefficient.

2. $u$, together with its derivatives, is bounded: there exists a constant $M_{1}>0$ such that

$$
\sup _{\Omega_{T}}\left(\left|\partial_{t} u\right|+|D u|+\left|D^{2} u\right|\right)+\sup _{\bar{\Omega}_{T}}|u| \leq M_{1}<\infty
$$

3. $g \geq 0$ and there exists a constant $M_{2}>0$ such that

$$
\sup _{\Omega_{T}}|\operatorname{div}(f)|<M_{2}<\infty .
$$

Under all the above assumptions, we have $u \geq 0$ in $\bar{\Omega}_{T}$.

Proof. We will argue by contradiction. Consider $v(t, x)=u(t, x) e^{-2 M_{2} t}$ and assume that there exists a constant $\delta>0$ such that

$$
\inf _{(t, x) \in \Omega_{T}} v(t, x)=-\delta<0 .
$$

Such a constant $\delta$ exists since by our assumption $v$ is bounded. It is not difficult to see that the infimum must be attained at some $\left(t^{*}, x^{*}\right) \in \bar{\Omega}_{T}$. If it were not true, then there exist $\left(t_{n}, x_{n}\right)$ becoming unbounded such that $v\left(t_{n}, x_{n}\right) \rightarrow-\delta$ as $n \rightarrow \infty$ which is a contradiction to our assumption that $u \in L^{p}\left(\Omega_{T}\right)$ and $u$ has bounded derivatives in $(t, x)$. It is evident that $0<t^{*} \leq T$. But then we compute

$$
\begin{aligned}
\left(\partial_{t} v\right)\left(t^{*}, x^{*}\right) & =-2 M_{2} v\left(t^{*}, x^{*}\right)+\left(\partial_{t} u\right)\left(t^{*}, x^{*}\right) e^{-2 M_{2} t^{*}} \\
& \geq\left(-2 M_{2}-\operatorname{div}(f)\right) v\left(t^{*}, x^{*}\right)-\nu\left((-\Delta)^{\alpha / 2} v\right)\left(t^{*}, x^{*}\right) .
\end{aligned}
$$


Since $v$ attains infimum at $\left(t^{*}, x^{*}\right)$, we have

$$
-\left((-\Delta)^{\alpha / 2} v\right)\left(t^{*}, x^{*}\right)=C_{\alpha} P . V . \int_{\mathbb{R}^{2}} \frac{v\left(t^{*}, y\right)-v\left(t^{*}, x^{*}\right)}{\left|y-x^{*}\right|^{2+\alpha}} d y \geq 0,
$$

where $C_{\alpha}$ is a positive constant. The integral representation (2.3) is valid since we are assuming $u$ is bounded and has bounded derivatives up to second order (in space). We now obtain

$$
\left(\partial_{t} v\right)\left(t^{*}, x^{*}\right) \geq M_{2} \delta>0 .
$$

But this is obviously a contradiction to the fact that $v$ attains its infimum at $\left(t^{*}, x^{*}\right)$. The lemma is proved.

The following pointwise estimate for fractional derivatives is proved in[13] and represents an improvement of an earlier estimate by Córdoba and Córdoba [11].

Lemma 2.8. (Pointwise estimate). Assume $f \in \mathcal{S}\left(\mathbb{R}^{2}\right), 0 \leq \alpha \leq 2$ and $\beta \geq-1$. Then the following pointwise inequality holds:

$$
|f(x)|^{\beta} f(x)(-\Delta)^{\alpha / 2} f(x) \geq \frac{1}{\beta+2}(-\Delta)^{\alpha / 2}|f(x)|^{\beta+2} .
$$

Proof. See Proposition 3.3 of [13].

\section{Local wellposedness and continuation criteria}

\subsection{Basic a priori estimates}

We first derive some a priori estimates needed later for the contraction argument. Throughout this subsection we assume $u \in C\left([0, \infty), L_{x}^{q}\left(\mathbb{R}^{2}\right) \cap\right.$ $\left.H_{x}^{s}\left(\mathbb{R}^{2}\right)\right), v \in C\left([0, \infty), L_{x}^{q}\left(\mathbb{R}^{2}\right) \cap H_{x}^{s}\left(\mathbb{R}^{2}\right)\right)$ for some $1<q<2$ and $s>3$ and they satisfy the equation

$$
\partial_{t} u+\nabla \cdot(u B(v))=-\nu(-\Delta)^{\frac{\alpha}{2}} u,
$$

where $\nu \geq 0$ and $0<\alpha<2$ are same parameters as in (1.1). Assume also that at $t=0$, we have

$$
u(0, x)=v(0, x)=u_{0}(x), \quad \forall x \in \mathbb{R}^{2},
$$

where $u_{0} \in H_{x}^{s}\left(\mathbb{R}^{2}\right) \cap L_{x}^{q}\left(\mathbb{R}^{2}\right)$ is a given function. We shall estimate the $L_{x}^{p}(q \leq p \leq \infty)$ and $H_{x}^{s}$ norms of $u$ in terms of those norms of $v$. Such estimates will be used later in the contraction argument (specifically we will be taking $u=u_{n+1}, v=u_{n}$, cf. (3.28)). To simplify the presentation, we divide the estimates into three steps. 
Step 1. $L_{x}^{p}$ estimate for $q \leq p \leq \infty$. Let $\epsilon>0$ be a small number. We first take $p$ such that $q \leq p<\infty$. Multiplying both sides of (3.1) by $|u|^{p-2} u \cdot e^{-\epsilon|x|}$ and integrating, we obtain

$$
\begin{aligned}
& \frac{1}{p} \frac{d}{d t} \int_{\mathbb{R}^{2}}|u|^{p} e^{-\epsilon|x|} d x=\int_{\mathbb{R}^{2}}|u|^{p-2} u \partial_{t} u e^{-\epsilon|x|} d x \\
& =-\int_{\mathbb{R}^{2}} \nabla \cdot(u B(v))|u|^{p-2} u e^{-\epsilon|x|} d x-\nu \int_{\mathbb{R}^{2}}(-\Delta)^{\alpha / 2} u|u|^{p-2} u e^{-\epsilon|x|} d x \\
& =-\int_{\mathbb{R}^{2}}(\nabla \cdot B(v))|u|^{p} e^{-\epsilon|x|} d x-\int_{\mathbb{R}^{2}} B(v) \cdot(\nabla u)|u|^{p-2} u e^{-\epsilon|x|} d x \\
& \quad-\nu \int_{\mathbb{R}^{2}}(-\Delta)^{\alpha / 2} u|u|^{p-2} u e^{-\epsilon|x|} d x \\
& =-\left(1-\frac{1}{p}\right) \int_{\mathbb{R}^{2}}(\nabla \cdot B(v))|u|^{p} e^{-\epsilon|x|} d x-\frac{\epsilon}{p} \int_{\mathbb{R}^{2}}\left(B(v) \cdot \frac{x}{|x|}\right)|u|^{p} e^{-\epsilon|x|} d x \\
& \quad-\nu \int_{\mathbb{R}^{2}}(-\Delta)^{\alpha / 2} u|u|^{p-2} u e^{-\epsilon|x|} d x \\
& \leq C \cdot\left(\|v\|_{L_{x}^{\infty}\left(\mathbb{R}^{2}\right)}+\epsilon\|B(v)\|_{L_{x}^{\infty}\left(\mathbb{R}^{2}\right)}\right) \int_{\mathbb{R}^{2}}|u|^{p} e^{-\epsilon|x|} d x \\
& \quad-\nu \int_{\mathbb{R}^{2}}(-\Delta)^{\alpha / 2} u|u|^{p-2} u e^{-\epsilon|x|} d x
\end{aligned}
$$

where the last inequality follows from the fact that $\nabla \cdot B(v)=$ Const $\cdot v$ (see (1.3)). $\mathrm{By}^{1}$ Lemma 2.8, choosing $\beta=p-2$, we have

$$
\begin{aligned}
-\int_{\mathbb{R}^{2}}(-\Delta)^{\alpha / 2} u|u|^{p-2} u e^{-\epsilon|x|} d x & \leq-\frac{1}{p} \int_{\mathbb{R}^{2}}(-\Delta)^{\alpha / 2}\left(|u|^{p}\right) e^{-\epsilon|x|} d x \\
& =-\frac{1}{p} \int_{\mathbb{R}^{2}}|u|^{p}(-\Delta)^{\alpha / 2}\left(e^{-\epsilon|x|}\right) d x .
\end{aligned}
$$

Now choose $r \in \mathbb{R}$ such that

$$
\frac{2}{\alpha}<r<\infty, \quad \text { if } 0<\alpha \leq 1
$$

and

$$
2 \leq r<\frac{2}{\alpha-1}, \quad \text { if } 1<\alpha<2 .
$$

We can then bound

$$
\begin{aligned}
\left\|(-\Delta)^{\alpha / 2}\left(e^{-\epsilon|x|}\right)\right\|_{L_{x}^{r}\left(\mathbb{R}^{2}\right)} & \leq \epsilon^{\alpha-\frac{2}{r}}\left\|(-\Delta)^{\alpha / 2}\left(e^{-|x|}\right)\right\|_{L_{x}^{r}\left(\mathbb{R}^{2}\right)} \\
& \leq \epsilon^{\alpha-\frac{2}{r}} \cdot \text { const } \cdot\left\|\frac{|\xi|^{\alpha}}{\left(1+|\xi|^{2}\right)^{\frac{3}{2}}}\right\|_{L_{\xi}^{\frac{r}{r-1}\left(\mathbb{R}^{2}\right)}} \leq C \epsilon^{\alpha-\frac{2}{r}}
\end{aligned}
$$

\footnotetext{
${ }^{1}$ Strictly speaking, in order to apply Lemma 2.8 , one needs to assume that $u \in \mathcal{S}\left(\mathbb{R}^{2}\right)$.
} But this can be easily fixed by a standard density argument. 
where $C$ is a constant depending on $(\alpha, r)$. It is not difficult to check that the last integral in $\xi$ converges due to our choice of the number $r$. By this last inequality and Hölder, we obtain

$$
\mid \text { RHS of }(3.4) \mid \leq \frac{C}{p}\|u\|_{L_{x}^{p-1}\left(\mathbb{R}^{2}\right)}^{p} \cdot \epsilon^{\alpha-\frac{2}{r}} .
$$

Plugging this estimate into (3.3) and integrating in time, we get

$$
\begin{aligned}
& \frac{1}{p} \int_{\mathbb{R}^{2}}|u(t)|^{p} e^{-\epsilon|x|} d x \leq \\
& \leq \frac{1}{p}\left\|u_{0}\right\|_{L_{x}^{p}\left(\mathbb{R}^{2}\right)}^{p}+C \int_{0}^{t}\left(\|v(\tau)\|_{L_{x}^{\infty}\left(\mathbb{R}^{2}\right)}+\epsilon\|B(v)(\tau)\|_{L_{x}^{\infty}\left(\mathbb{R}^{2}\right)}\right)\|u(\tau)\|_{L_{x}^{p}\left(\mathbb{R}^{2}\right)}^{p} d \tau \\
& \quad+\nu \frac{C}{p} \int_{0}^{t}\|u(\tau)\|_{L_{x}^{p}}^{p} d \tau \epsilon^{\alpha-\frac{2}{r}} .
\end{aligned}
$$

Now since $\|B(v)\|_{L_{x}^{\infty}\left(\mathbb{R}^{2}\right)} \lesssim\|v\|_{L_{x}^{q}\left(\mathbb{R}^{2}\right)}+\|v\|_{L_{x}^{\infty}\left(\mathbb{R}^{2}\right)}$ and therefore is bounded, taking $\epsilon \rightarrow 0$ gives us

$$
\frac{1}{p}\|u(t)\|_{L_{x}^{p}\left(\mathbb{R}^{2}\right)}^{p} \leq \frac{1}{p}\left\|u_{0}\right\|_{L_{x}^{p}\left(\mathbb{R}^{2}\right)}^{p}+C \int_{0}^{t}\|v(\tau)\|_{L_{x}^{\infty}} \cdot\|u(\tau)\|_{L_{x}^{p}\left(\mathbb{R}^{2}\right)}^{p} d \tau .
$$

Gronwall then yields

$$
\|u(t)\|_{L_{x}^{p}\left(\mathbb{R}^{2}\right)} \leq\left\|u_{0}\right\|_{L_{x}^{p}\left(\mathbb{R}^{2}\right)} \cdot \exp \left(C \int_{0}^{t}\|v(\tau)\|_{L_{x}^{\infty}\left(\mathbb{R}^{2}\right)} d \tau\right) .
$$

This is the first estimate we need. Now taking $p \rightarrow \infty$ in (3.6) gives us

$$
\|u(t)\|_{L_{x}^{\infty}\left(\mathbb{R}^{2}\right)} \leq\left\|u_{0}\right\|_{L_{x}^{\infty}\left(\mathbb{R}^{2}\right)} \cdot \exp \left(C \int_{0}^{t}\|v(\tau)\|_{L_{x}^{\infty}\left(\mathbb{R}^{2}\right)} d \tau\right) .
$$

Now assume $T>0$ is such that

$$
T \cdot \max _{0 \leq t \leq T}\|v(t)\|_{L_{x}^{\infty}} \leq \frac{\log 2}{C},
$$

then clearly

$$
\exp \left(C \int_{0}^{T}\|v(\tau)\|_{L_{x}^{\infty}\left(\mathbb{R}^{2}\right)} d \tau\right) \leq 2 .
$$

Plugging this estimate back into (3.7) and we obtain

$$
\sup _{0 \leq t \leq T}\|u(t)\|_{L_{x}^{\infty}\left(\mathbb{R}^{2}\right)} \leq 2\left\|u_{0}\right\|_{L_{x}^{\infty}\left(\mathbb{R}^{2}\right)} .
$$

Using the last two estimates and (3.6), we finally get

$$
\sup _{0 \leq t \leq T}\|u(t)\|_{L_{x}^{p}\left(\mathbb{R}^{2}\right)} \leq 2\left\|u_{0}\right\|_{L_{x}^{p}\left(\mathbb{R}^{2}\right)}, \quad \forall q \leq p \leq \infty .
$$

This concludes the $L_{x}^{p}$ estimate. 
Remark 3.2. In the $L_{x}^{p}$ estimate above, we need to use the function $e^{-\epsilon|x|}$ as a cut-off to make the integral containing the diffusion term converge. More precisely, for $1<q<2$, the integral

$$
\int_{\mathbb{R}^{2}}(-\Delta)^{\alpha / 2} u|u|^{q-2} u d x
$$

may not converge since we are only assuming that $u \in H_{x}^{s}\left(\mathbb{R}^{2}\right) \cap L_{x}^{q}\left(\mathbb{R}^{2}\right)$. This is the reason why we use this regularization instead of multiplying directly by $|u|^{p-2} u$.

Step 2. $\dot{H}_{x}^{s}$ estimate for $s>0$. Since the low frequency part of the $\dot{H}_{x}^{s}$ norm of the function $u$ can be readily controlled by its $L_{x}^{q}(q \leq 2)$ norm, it suffices for us to derive a priori estimates for the high frequency part of the $\dot{H}_{x}^{s}$ norm of the function $u$, i. e., the quantity

$$
\|u(t)\|_{Y^{s}\left(\mathbb{R}^{2}\right)}^{2}:=\sum_{k>0} 2^{2 k s}\left\|P_{k} u(t)\right\|_{L_{x}^{2}\left(\mathbb{R}^{2}\right)}^{2}
$$

Applying the projector $P_{k}$ to both sides of (3.1), multiplying by $P_{k} u$ and integrating, we have

$$
\begin{aligned}
\frac{1}{2} \frac{d}{d t}\left\|P_{k} u\right\|_{L_{x}^{2}\left(\mathbb{R}^{2}\right)}^{2}+\nu\left\|(-\Delta)^{\alpha / 4} P_{k} u\right\|_{L_{x}^{2}\left(\mathbb{R}^{2}\right)}^{2} & = \\
& =-\int_{\mathbb{R}^{2}} P_{k} \nabla \cdot(u B(v)) P_{k} u d x .
\end{aligned}
$$

By Lemma 2.4, we have the bound

$$
\begin{aligned}
\sum_{k>0} 2^{2 k s} \mid \int_{\mathbb{R}^{2}} P_{k} \nabla & (u B(v)) P_{k} u d x \mid \lesssim \\
\lesssim( & \left.\|u\|_{L_{x}^{\infty}\left(\mathbb{R}^{2}\right)}+\|D B(v)\|_{L_{x}^{\infty}\left(\mathbb{R}^{2}\right)}\right)\left\|P_{>-20} u\right\|_{\dot{H}_{x}^{s}\left(\mathbb{R}^{2}\right)}^{2} \\
& +\|u\|_{L_{x}^{\infty}\left(\mathbb{R}^{2}\right)}\left\|P_{>-20} B(v)\right\|_{\dot{H}_{x}^{s+1}\left(\mathbb{R}^{2}\right)}^{2} \\
\lesssim( & \left.\|u\|_{L_{x}^{\infty}\left(\mathbb{R}^{2}\right)}+\|D B(v)\|_{L_{x}^{\infty}\left(\mathbb{R}^{2}\right)}\right)\left\|P_{>-20} u\right\|_{\dot{H}_{x}^{s}\left(\mathbb{R}^{2}\right)}^{2} \\
& +\|u\|_{L_{x}^{\infty}\left(\mathbb{R}^{2}\right)}\left\|P_{>-20} v\right\|_{\dot{H}_{x}^{s}\left(\mathbb{R}^{2}\right)}^{2},
\end{aligned}
$$

where the last inequality follows from (1.3) and the fact that the Riesz operator $|\nabla|^{-1} \nabla$ is bounded on $L_{x}^{2}\left(\mathbb{R}^{2}\right)$ :

$$
\left\|P_{>-20} B(v)\right\|_{\dot{H}_{x}^{s+1}\left(\mathbb{R}^{2}\right)} \lesssim\left\|P_{>-20}|\nabla|^{s} v\right\|_{L_{x}^{2}\left(\mathbb{R}^{2}\right)} \lesssim\left\|P_{>-20} v\right\|_{\dot{H}_{x}^{s}\left(\mathbb{R}^{2}\right)} .
$$


Therefore by (3.10), (3.11) and (3.12), we obtain

$$
\begin{gathered}
\frac{1}{2} \frac{d}{d t}\|u(t)\|_{Y^{s}\left(\mathbb{R}^{2}\right)}^{2}+\nu \sum_{k>0} 2^{2 k s}\left\|P_{k}(-\Delta)^{\alpha / 4} u\right\|_{L_{x}^{2}\left(\mathbb{R}^{2}\right)}^{2} \lesssim \\
\lesssim\left(\|u\|_{L_{x}^{\infty}\left(\mathbb{R}^{2}\right)}+\|D B(v)\|_{L_{x}^{\infty}\left(\mathbb{R}^{2}\right)}\right)\left\|P_{>-20} u\right\|_{\dot{H}_{x}^{s}\left(\mathbb{R}^{2}\right)}^{2} \\
+\|u\|_{L_{x}^{\infty}\left(\mathbb{R}^{2}\right)}\left\|P_{>-20} v\right\|_{\dot{H}_{x}^{s}\left(\mathbb{R}^{2}\right)}^{2}
\end{gathered}
$$

Step 3. Conclusion of the estimates. Taking $s=2$ in (3.13) and using Lemma 2.5, we have

$$
\begin{aligned}
\frac{d}{d t}\|u(t)\|_{Y^{2}\left(\mathbb{R}^{2}\right)}^{2} \lesssim & \left(\|u(t)\|_{L_{x}^{\infty}\left(\mathbb{R}^{2}\right)}+\|D B(v)\|_{L_{x}^{\infty}\left(\mathbb{R}^{2}\right)}\right) \cdot \\
& \cdot\left(\|u(t)\|_{L_{x}^{q}\left(\mathbb{R}^{2}\right)}^{2}+\|u(t)\|_{Y^{2}\left(\mathbb{R}^{2}\right)}^{2}\right)+\|u\|_{L_{x}^{\infty}\left(\mathbb{R}^{2}\right)}\left\|P_{>-20} v\right\|_{\dot{H}_{x}^{2}\left(\mathbb{R}^{2}\right)}^{2} \\
\lesssim & \left(\|u(t)\|_{L_{x}^{\infty}\left(\mathbb{R}^{2}\right)}+\|v(t)\|_{L_{x}^{q}\left(\mathbb{R}^{2}\right)}+\|v(t)\|_{Y^{2}\left(\mathbb{R}^{2}\right)}\right) \cdot \\
& \cdot\left(\|u(t)\|_{L_{x}^{q}\left(\mathbb{R}^{2}\right)}^{2}+\|u(t)\|_{Y^{2}\left(\mathbb{R}^{2}\right)}^{2}\right)+\|u\|_{L_{x}^{\infty}\left(\mathbb{R}^{2}\right)}\left\|P_{>-20} v\right\|_{\dot{H}_{x}^{2}\left(\mathbb{R}^{2}\right)}^{2}
\end{aligned}
$$

By (3.9) and choosing $T$ satisfying (3.8), we then have

$$
\begin{aligned}
\frac{d}{d t}\|u(t)\|_{Y^{2}\left(\mathbb{R}^{2}\right) \lesssim}^{2} & \left(2\left\|u_{0}\right\|_{L_{x}^{\infty}\left(\mathbb{R}^{2}\right)}+\|v(t)\|_{Y^{2}\left(\mathbb{R}^{2}\right)}+\|v(t)\|_{L_{x}^{q}\left(\mathbb{R}^{2}\right)}\right) \cdot \\
& \cdot\left(4\left\|u_{0}\right\|_{L_{x}^{q}\left(\mathbb{R}^{2}\right)}^{2}+\|u(t)\|_{Y^{2}\left(\mathbb{R}^{2}\right)}^{2}\right)+2\left\|u_{0}\right\|_{L_{x}^{\infty}\left(\mathbb{R}^{2}\right)}\left\|P_{>-20} v\right\|_{\dot{H}_{x}^{2}\left(\mathbb{R}^{2}\right)}^{2} \\
\lesssim & \left(\left\|u_{0}\right\|_{L_{x}^{q}\left(\mathbb{R}^{2}\right)}+\left\|u_{0}\right\|_{Y^{2}\left(\mathbb{R}^{2}\right)}+\|v(t)\|_{Y^{2}\left(\mathbb{R}^{2}\right)}+\|v(t)\|_{L_{x}^{q}\left(\mathbb{R}^{2}\right)}\right) \cdot \\
& \cdot\left(\left\|u_{0}\right\|_{L_{x}^{q}\left(\mathbb{R}^{2}\right)}^{2}+\|u(t)\|_{Y^{2}\left(\mathbb{R}^{2}\right)}^{2}\right)+\left\|u_{0}\right\|_{H_{x}^{2}\left(\mathbb{R}^{2}\right)}\left\|P_{>-20} v\right\|_{\dot{H}_{x}^{2}\left(\mathbb{R}^{2}\right)}^{2},
\end{aligned}
$$

where the last inequality follows from Lemma 2.5 and Sobolev embedding. Now choose $T$ such that

1. $T$ satisfies (3.8).

2. The $Y^{2}$ and $L_{x}^{q}$ norm of $v$ on $[0, T]$ is not too large:

$$
\max _{0 \leq t \leq T}\left(\|v(t)\|_{Y^{2}\left(\mathbb{R}^{2}\right)}+\|v(t)\|_{L_{x}^{q}\left(\mathbb{R}^{2}\right)}\right) \leq 4 \cdot\left(\left\|u_{0}\right\|_{Y^{2}\left(\mathbb{R}^{2}\right)}+\left\|u_{0}\right\|_{L_{x}^{q}\left(\mathbb{R}^{2}\right)}\right) .
$$

3. $T$ also satisfies

$$
T \leq \frac{\tilde{C}_{1}}{\left\|u_{0}\right\|_{H_{x}^{2}\left(\mathbb{R}^{2}\right)}+\left\|u_{0}\right\|_{L_{x}^{q}\left(\mathbb{R}^{2}\right)}},
$$

where the constant $\tilde{C}_{1}$ depends only on $q, \nu, \alpha$ and will later be made suitably small. 
Then for any $t \leq T$, we have

RHS of $(3.15) \lesssim$

$$
\begin{aligned}
& \lesssim\left(\left\|u_{0}\right\|_{L_{x}^{q}\left(\mathbb{R}^{2}\right)}+\left\|u_{0}\right\|_{Y^{2}\left(\mathbb{R}^{2}\right)}\right) \cdot\left(\left\|u_{0}\right\|_{L_{x}^{q}\left(\mathbb{R}^{2}\right)}^{2}+\|u(t)\|_{Y^{2}\left(\mathbb{R}^{2}\right)}^{2}\right) \\
& \quad+\left(\left\|u_{0}\right\|_{L_{x}^{q}\left(\mathbb{R}^{2}\right)}+\left\|u_{0}\right\|_{Y^{2}\left(\mathbb{R}^{2}\right)}\right)^{3} \\
& \lesssim\left(\left\|u_{0}\right\|_{L_{x}^{q}\left(\mathbb{R}^{2}\right)}+\left\|u_{0}\right\|_{Y^{2}\left(\mathbb{R}^{2}\right)}\right)\|u(t)\|_{Y^{2}\left(\mathbb{R}^{2}\right)}^{2}+\left(\left\|u_{0}\right\|_{L_{x}^{q}\left(\mathbb{R}^{2}\right)}+\left\|u_{0}\right\|_{Y^{2}\left(\mathbb{R}^{2}\right)}\right)^{3} .
\end{aligned}
$$

By the assumptions on $T,(3.17)$, choosing $\tilde{C}_{1}$ sufficiently small , a simple Gronwall argument yields

$$
\sup _{0 \leq t \leq T}\|u(t)\|_{Y^{2}\left(\mathbb{R}^{2}\right)} \leq 2 \cdot\left(\left\|u_{0}\right\|_{L_{x}^{q}\left(\mathbb{R}^{2}\right)}+\left\|u_{0}\right\|_{Y^{2}\left(\mathbb{R}^{2}\right)}\right) .
$$

By (3.9), this implies

$$
\sup _{0 \leq t \leq T}\left(\|u(t)\|_{L_{x}^{q}\left(\mathbb{R}^{2}\right)}+\|u(t)\|_{Y^{2}\left(\mathbb{R}^{2}\right)}\right) \leq \leq 4 \cdot\left(\left\|u_{0}\right\|_{Y^{2}\left(\mathbb{R}^{2}\right)}+\left\|u_{0}\right\|_{L_{x}^{q}\left(\mathbb{R}^{2}\right)}\right) .
$$

This ends the estimate for $s=2$. By the assumptions on $T$, (3.8), (3.16) and Lemma 2.5, it is not difficult to see that

$$
\begin{aligned}
& \sup _{0 \leq t \leq T}\left(\|u(t)\|_{L_{x}^{\infty}\left(\mathbb{R}^{2}\right)}+\|D B(v(t))\|_{L_{x}^{\infty}\left(\mathbb{R}^{2}\right)}\right) \leq \\
& \quad \leq \tilde{C}_{2} \cdot\left(\left\|u_{0}\right\|_{Y^{2}\left(\mathbb{R}^{2}\right)}+\left\|u_{0}\right\|_{L_{x}^{q}\left(\mathbb{R}^{2}\right)}\right),
\end{aligned}
$$

where $\tilde{C}_{2}$ is a constant depending on $q$. Plugging the estimate (3.20) into (3.13), we obtain for any $t \leq T$ and $s \geq 2$ :

$$
\begin{aligned}
\frac{1}{2} \frac{d}{d t}\|u(t)\|_{Y^{s}\left(\mathbb{R}^{2}\right)}^{2} \lesssim( & \left.\left\|u_{0}\right\|_{Y^{2}\left(\mathbb{R}^{2}\right)}+\left\|u_{0}\right\|_{L_{x}^{q}\left(\mathbb{R}^{2}\right)}\right) \cdot\left\|P_{>-20} u(t)\right\|_{\dot{H}_{x}^{s}\left(\mathbb{R}^{2}\right)}^{2} \\
& +\left\|u_{0}\right\|_{L_{x}^{\infty}\left(\mathbb{R}^{2}\right)} \cdot\|v(t)\|_{H_{x}^{s}\left(\mathbb{R}^{2}\right)}^{2} \\
\lesssim( & \left.\left\|u_{0}\right\|_{Y^{2}\left(\mathbb{R}^{2}\right)}+\left\|u_{0}\right\|_{L_{x}^{q}\left(\mathbb{R}^{2}\right)}\right) \cdot\left(\|u(t)\|_{Y^{s}\left(\mathbb{R}^{2}\right)}^{2}+\|v(t)\|_{Y^{s}\left(\mathbb{R}^{2}\right)}^{2}\right) \\
& +\left(\left\|u_{0}\right\|_{Y^{2}\left(\mathbb{R}^{2}\right)}+\left\|u_{0}\right\|_{L_{x}^{q}\left(\mathbb{R}^{2}\right)}\right)^{3} .
\end{aligned}
$$

Now a simple Gronwall argument gives that for any $t \leq T$,

$$
\begin{aligned}
\|u(t)\|_{Y^{s}\left(\mathbb{R}^{2}\right)}^{2} \leq & e^{\tilde{C}_{3} t \cdot\left(\left\|u_{0}\right\|_{Y^{s}\left(\mathbb{R}^{2}\right)}+\left\|u_{0}\right\|_{L_{x}^{q}\left(\mathbb{R}^{2}\right)}\right)} . \\
& \cdot\left(\left\|u_{0}\right\|_{Y^{s}\left(\mathbb{R}^{2}\right)}^{2}+2 t \cdot\left(\left\|u_{0}\right\|_{Y^{2}\left(\mathbb{R}^{2}\right)}+\left\|u_{0}\right\|_{L_{x}^{q}\left(\mathbb{R}^{2}\right)}\right)^{3}\right. \\
+ & \left.2 t\left(\left\|u_{0}\right\|_{Y^{2}\left(\mathbb{R}^{2}\right)}+\left\|u_{0}\right\|_{L_{x}^{q}\left(\mathbb{R}^{2}\right)}\right) \cdot \max _{0 \leq \tau \leq t}\|v(\tau)\|_{Y^{s}\left(\mathbb{R}^{2}\right)}^{2}\right),
\end{aligned}
$$

where $\tilde{C}_{3}$ is a constant depending on the numbers $(s, q)$. 
Choosing $T$ as in (3.17) and letting the constant $\tilde{C}_{1}$ be sufficiently small, we then have

$$
\begin{aligned}
& \max _{0 \leq t \leq T}\|u(t)\|_{Y^{2}\left(\mathbb{R}^{2}\right)}^{2} \leq \\
& \quad \leq 2\left\|u_{0}\right\|_{Y^{s}\left(\mathbb{R}^{2}\right)}^{2}+\frac{1}{2}\left(\left\|u_{0}\right\|_{Y^{2}\left(\mathbb{R}^{2}\right)}+\left\|u_{0}\right\|_{L_{x}^{q}\left(\mathbb{R}^{2}\right)}\right)^{2}+\frac{1}{2} \cdot \max _{0 \leq t \leq T}\|v(t)\|_{Y^{s}\left(\mathbb{R}^{2}\right)}^{2} \\
& \quad \leq 2\left\|u_{0}\right\|_{Y^{s}\left(\mathbb{R}^{2}\right)}^{2}+\left\|u_{0}\right\|_{Y^{2}\left(\mathbb{R}^{2}\right)}^{2}+\left\|u_{0}\right\|_{L_{x}^{q}\left(\mathbb{R}^{2}\right)}^{2}+\frac{1}{2} \cdot \max _{0 \leq t \leq T}\|v(t)\|_{Y^{s}\left(\mathbb{R}^{2}\right)}^{2} .
\end{aligned}
$$

Now if we assume that

$$
\max _{0 \leq t \leq T}\|v(t)\|_{Y^{s}\left(\mathbb{R}^{2}\right)}^{2} \leq 4\left\|u_{0}\right\|_{Y^{s}\left(\mathbb{R}^{2}\right)}^{2}+2\left\|u_{0}\right\|_{Y^{2}\left(\mathbb{R}^{2}\right)}^{2}+2\left\|u_{0}\right\|_{L_{x}^{q}\left(\mathbb{R}^{2}\right)}^{2},
$$

then we obtain

$$
\max _{0 \leq t \leq T}\|u(t)\|_{Y^{s}\left(\mathbb{R}^{2}\right)}^{2} \leq 4\left\|u_{0}\right\|_{Y^{s}\left(\mathbb{R}^{2}\right)}^{2}+2\left\|u_{0}\right\|_{Y^{2}\left(\mathbb{R}^{2}\right)}^{2}+2\left\|u_{0}\right\|_{L_{x}^{q}\left(\mathbb{R}^{2}\right)}^{2},
$$

Next plugging this estimate together with (3.16), (3.9) and (3.20) into (3.13), we obtain for some constant $\tilde{C}_{4}>0$ depending on $\left(\left\|u_{0}\right\|_{H_{x}^{s}\left(\mathbb{R}^{2}\right)},\left\|u_{0}\right\|_{L_{x}^{q}\left(\mathbb{R}^{2}\right)}\right)$,

$$
\frac{1}{2} \frac{d}{d t}\|u(t)\|_{Y^{s}\left(\mathbb{R}^{2}\right)}^{2}+\nu\|u(t)\|_{H_{x}^{s+\frac{\alpha}{2}}\left(\mathbb{R}^{2}\right)}^{2} \leq \tilde{C}_{4}
$$

where we have used the fact that the low frequency part of the $H_{x}^{s+\frac{\alpha}{2}}\left(\mathbb{R}^{2}\right)$ and $H_{x}^{s}\left(\mathbb{R}^{2}\right)$ norms of $u$ can be controlled by its $L_{x}^{q}\left(\mathbb{R}^{2}\right)$-norm which in turn is bounded by (3.9). Integrating on $[0, T]$ and we get

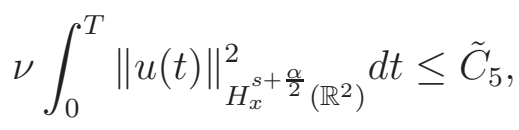

where $\tilde{C}_{5}$ is another constant depending on $\left(\left\|u_{0}\right\|_{H_{x}^{s}\left(\mathbb{R}^{2}\right)},\left\|u_{0}\right\|_{L_{x}^{q}\left(\mathbb{R}^{2}\right)}\right)$.

Finally, we summarize the basic a priori estimates as follows. There exist a constant $\tilde{c}_{1}>0$ depending on the numbers $(q, s)$ such that if $T$ satisfies the following conditions:

(i) The $L_{x}^{q}$ and $Y^{2}$ norms of $v$ are not too large:

$$
\text { (3.21) } \max _{0 \leq t \leq T}\left(\|v(t)\|_{L_{x}^{q}\left(\mathbb{R}^{2}\right)}+\|v(t)\|_{Y^{2}\left(\mathbb{R}^{2}\right)}\right) \leq 4 \cdot\left(\left\|u_{0}\right\|_{L_{x}^{q}\left(\mathbb{R}^{2}\right)}+\left\|u_{0}\right\|_{Y^{2}\left(\mathbb{R}^{2}\right)}\right) ;
$$

(ii) The $Y^{s}$ norm of $v$ is not too large:

$$
\max _{0 \leq t \leq T}\|v(t)\|_{Y^{s}\left(\mathbb{R}^{2}\right)}^{2} \leq 4\left\|u_{0}\right\|_{Y^{s}\left(\mathbb{R}^{2}\right)}^{2}+2\left\|u_{0}\right\|_{Y^{2}\left(\mathbb{R}^{2}\right)}^{2}+2\left\|u_{0}\right\|_{L_{x}^{q}\left(\mathbb{R}^{2}\right)}^{2}
$$


(iii) $T$ satisfies the bound ${ }^{2}$ :

$$
T \leq \frac{\tilde{c}_{1}}{\left\|u_{0}\right\|_{H_{x}^{2}\left(\mathbb{R}^{2}\right)}+\left\|u_{0}\right\|_{L_{x}^{q}\left(\mathbb{R}^{2}\right)}}
$$

then for the function $u$ we have the following:

(a) The $L_{x}^{q}$ and $Y^{2}$ norms of $u$ are not too large:

$$
\begin{aligned}
& \max _{0 \leq t \leq T}\left(\|u(t)\|_{L_{x}^{q}\left(\mathbb{R}^{2}\right)}+\|u(t)\|_{Y^{2}\left(\mathbb{R}^{2}\right)}\right) \leq \\
& \quad \leq 4 \cdot\left(\left\|u_{0}\right\|_{L_{x}^{q}\left(\mathbb{R}^{2}\right)}+\left\|u_{0}\right\|_{Y^{2}\left(\mathbb{R}^{2}\right)}\right) ;
\end{aligned}
$$

(b) The $Y^{s}$ norm of $u$ is not too large:

$$
\max _{0 \leq t \leq T}\|u(t)\|_{Y^{s}\left(\mathbb{R}^{2}\right)}^{2} \leq 4\left\|u_{0}\right\|_{Y^{s}\left(\mathbb{R}^{2}\right)}^{2}+2\left\|u_{0}\right\|_{Y^{2}\left(\mathbb{R}^{2}\right)}^{2}+2\left\|u_{0}\right\|_{L_{x}^{q}\left(\mathbb{R}^{2}\right)}^{2}
$$

(c) The following bound also holds:

$$
\sup _{0 \leq t \leq T}\|u(t)\|_{H_{x}^{s}\left(\mathbb{R}^{2}\right)}+\nu \int_{0}^{T}\|u(t)\|_{H_{x}^{s+\frac{\alpha}{2}}\left(\mathbb{R}^{2}\right)}^{2} d t \leq \tilde{D}_{1}
$$

(d) For any $t \leq T$,

$$
\|u(t)\|_{Y^{s}\left(\mathbb{R}^{2}\right)} \leq e^{\tilde{D}_{2} t}\left(\left\|u_{0}\right\|_{Y^{s}\left(\mathbb{R}^{2}\right)}+\tilde{D}_{3} t\right) .
$$

Here $\tilde{D}_{1}, \tilde{D}_{2}$ and $\tilde{D}_{3}$ are positive constants depending on $\left\|u_{0}\right\|_{H_{x}^{s}\left(\mathbb{R}^{2}\right)}$ and $\left\|u_{0}\right\|_{L_{x}^{q}\left(\mathbb{R}^{2}\right)}$. Note that for the control of the $Y^{s}$-(semi)norm of $u$, the estimate (3.27) is slightly better than the mere boundedness in (3.26). We shall need (3.27) later to show the continuity of $u$ in $H^{s}$-norm at $t=0$ (see (3.38)).

Remark 3.3. As indicated before the above estimates will be used in the contraction argument. We remark that (a) and (b) above ((3.24) and (3.25)) are the same estimates as the ones assumed for $v,(3.21)$ and (3.22). This will be required later to guarantee that the estimates obtained for the sequence of approximate solutions $u_{n}$ are uniform in $n$.

\footnotetext{
${ }^{2}$ Note here by the Sobolev embedding inequality $\|f\|_{L_{x}^{\infty}\left(\mathbb{R}^{2}\right)} \leq\|f\|_{H_{x}^{2}\left(\mathbb{R}^{2}\right)}$, the condition (3.8) can be taken care of by making $\tilde{c}_{1}$ sufficiently small.
} 


\subsection{Contraction arguments}

We assume that the initial data $u_{0} \in L_{x}^{q}\left(\mathbb{R}^{2}\right) \cap H_{x}^{s}\left(\mathbb{R}^{2}\right)$ for some $1<q<2$ and $s>3$. We will construct a sequence of functions $\left\{u_{k}\right\}_{k=1}^{\infty}$ in the following manner. Define $u_{1}(t, x)=u_{0}(x)$. We define $u_{n+1}(n \geq 1)$ as the solution of

$$
\left\{\begin{array}{l}
\partial_{t} u_{n+1}+\nabla \cdot\left(u_{n+1} B\left(u_{n}\right)\right)=-\nu(-\Delta)^{\alpha / 2} u_{n+1}, \\
u_{n+1}(0)=u_{0} .
\end{array}\right.
$$

Since we are assuming high regularity on the initial data, no regularization is needed in this approximation scheme.

Step 1: Properties of solutions to the linear problem. For all $n \geq 1$, we have $u_{n} \in C\left([0, \infty), H_{x}^{s}\left(\mathbb{R}^{2}\right) \cap L_{x}^{q}\left(\mathbb{R}^{2}\right)\right)$. This can be shown by an induction on $n$. The claim obviously holds for $n=1$ since $u_{1}(t, x) \equiv u_{0}$ and $u_{0} \in$ $H_{x}^{s}\left(\mathbb{R}^{2}\right) \cap L_{x}^{q}\left(\mathbb{R}^{2}\right)$. Assume the statement is true for $u_{n}$ with $n \geq 1$. Then for $u_{n+1}$, using the fact that $u_{n} \in C\left([0, \infty), H_{x}^{s}\left(\mathbb{R}^{2}\right) \cap L_{x}^{q}\left(\mathbb{R}^{2}\right)\right)$, it is not difficult to show that $(3.28)$ has a unique solution in $C\left([0, \infty), H_{x}^{s}\left(\mathbb{R}^{2}\right)\right)$. To show $u_{n+1} \in C\left([0, \infty), L_{x}^{q}\left(\mathbb{R}^{2}\right)\right)$, we use (3.28) and Duhamel's formula to write $u_{n+1}$ as

$$
u_{n+1}(t)=e^{-\nu t(-\Delta)^{\alpha / 2}} u_{0}-\int_{0}^{t} e^{-\nu(t-\tau)(-\Delta)^{\alpha / 2}} \nabla \cdot\left(u_{n+1}(\tau) B\left(u_{n}\right)(\tau)\right) d \tau .
$$

We note that the above expression for $u_{n+1}$, with the obvious interpretation, can be used for the case $\nu=0$.

Now use the fact that

$$
\left\|e^{-\nu t(-\Delta)^{\alpha / 2}} f\right\|_{L_{x}^{p}\left(\mathbb{R}^{2}\right)} \lesssim\|f\|_{L_{x}^{p}\left(\mathbb{R}^{2}\right)}
$$

for any $t \geq 0,1 \leq p \leq \infty$, we quickly obtain

$$
\begin{gathered}
\left\|u_{n+1}(t)\right\|_{L_{x}^{q}\left(\mathbb{R}^{2}\right)} \lesssim\left\|u_{0}\right\|_{L_{x}^{q}\left(\mathbb{R}^{2}\right)}+\int_{0}^{t}\left\|\nabla \cdot\left(u_{n+1}(\tau) B\left(u_{n}\right)(\tau)\right)\right\|_{L_{x}^{q}\left(\mathbb{R}^{2}\right)} d \tau \\
\lesssim\left\|u_{0}\right\|_{L_{x}^{q}\left(\mathbb{R}^{2}\right)}+\int_{0}^{t}\left(\left\|\nabla u_{n+1}(\tau)\right\|_{L_{x}^{2}\left(\mathbb{R}^{2}\right)} \cdot\left\|(-\Delta)^{-1} \nabla u_{n}(\tau)\right\|_{L_{x}^{2 q}\left(\frac{2 q}{2-q}\left(\mathbb{R}^{2}\right)\right.}\right. \\
\left.\quad+\left\|u_{n+1}(\tau)\right\|_{L_{x}^{\infty}\left(\mathbb{R}^{2}\right)}\left\|u_{n}(\tau)\right\|_{L_{x}^{q}\left(\mathbb{R}^{2}\right)}\right) d \tau \\
\lesssim\left\|u_{0}\right\|_{L_{x}^{q}\left(\mathbb{R}^{2}\right)}+\int_{0}^{t}\left\|u_{n+1}(\tau)\right\|_{H_{x}^{s}\left(\mathbb{R}^{2}\right)}\left\|u_{n}(\tau)\right\|_{L_{x}^{q}\left(\mathbb{R}^{2}\right)} d \tau
\end{gathered}
$$

where the last inequality follows from Sobolev embedding. This estimate shows that the $L_{x}^{q}$ norm of $u_{n+1}$ is bounded on any finite time interval. 
By a similar argument we can show the strong continuity (at any finite $t$ including right continuity at $t=0$ ) of $u_{n+1}$. Therefore we conclude $u_{n+1} \in$ $C\left([0, \infty), L_{x}^{q}\left(\mathbb{R}^{2}\right)\right)$.

Step 2: Uniform estimates on the sequence $u_{n}$. We claim that there exists a constant $c_{1}>0$ depending only on $(\alpha, q, \nu)$, such that if

$$
0<T \leq \frac{c_{1}}{\left\|u_{0}\right\|_{H_{x}^{2}\left(\mathbb{R}^{2}\right)}+\left\|u_{0}\right\|_{L_{x}^{q}\left(\mathbb{R}^{2}\right)}}
$$

then for any $s \geq 2, q \leq p \leq \infty, n \geq 1$, we have

$$
\sup _{0 \leq t \leq T}\left(\left\|u_{n}(t)\right\|_{L_{x}^{p}\left(\mathbb{R}^{2}\right)}+\left\|u_{n}(t)\right\|_{H_{x}^{s}\left(\mathbb{R}^{2}\right)}\right)+\nu \int_{0}^{T}\left\|u_{n}(t)\right\|_{H_{x}^{s+\frac{\alpha}{2}}\left(\mathbb{R}^{2}\right)}^{2} d t \leq D_{1}
$$

and for any $t \leq T$,

$$
\left\|u_{n}(t)\right\|_{Y^{s}\left(\mathbb{R}^{2}\right)} \leq e^{D_{2} t}\left(\left\|u_{0}\right\|_{Y^{s}\left(\mathbb{R}^{2}\right)}+D_{3} t\right) .
$$

Here $D_{1}, D_{2}$ and $D_{3}$ are positive constants depending on $\left\|u_{0}\right\|_{H_{x}^{s}\left(\mathbb{R}^{2}\right)}$ and $\left\|u_{0}\right\|_{L_{x}^{q}\left(\mathbb{R}^{2}\right)}$. This claim can be proved using the a priori estimates derived earlier and an induction on $n$. Specifically take $T>0$ such that

$$
T \leq \frac{\tilde{c}_{1}}{\left\|u_{0}\right\|_{H_{x}^{2}\left(\mathbb{R}^{2}\right)}+\left\|u_{0}\right\|_{L_{x}^{q}\left(\mathbb{R}^{2}\right)}}
$$

where $\tilde{c}_{1}$ is the same as in (3.23). Then for $n=1$ the claim obviously holds since $u_{1}(t, x) \equiv u_{0}$. For $n \geq 2$, the claim also holds by induction (note that as pointed out in remark 3.3 the estimates (3.21) and (3.24), (3.22) and (3.25) are consistent with each other). We leave the details to the interested reader.

Step 3: Contraction in $C\left(\left[0, T^{\prime}\right], L_{x}^{q}\left(\mathbb{R}^{2}\right)\right)$ for some $T^{\prime} \leq T$. Here we need to show the convergence of the whole sequence in order to pass to the limit in (3.28). Denote $w_{n+1}=u_{n+1}-u_{n}$. Using (3.28), a direct calculation shows that $w_{n+1}$ satisfies

$$
\partial_{t} w_{n+1}+\nabla \cdot\left(w_{n+1} B\left(u_{n}\right)\right)+\nabla \cdot\left(u_{n} B\left(w_{n}\right)\right)=-\nu(-\Delta)^{\alpha / 2} w_{n+1}
$$

Let $\epsilon>0$ be a small number which we will send to 0 later. Multiplying both 
sides of (3.32) by $\left|w_{n+1}\right|^{q-2} w_{n+1} e^{-\epsilon|x|}$ and integrating, we obtain

$$
\begin{aligned}
& \frac{1}{q} \frac{d}{d t} \int_{\mathbb{R}^{2}}\left|w_{n+1}\right|^{q} e^{-\epsilon|x|} d x=\int_{\mathbb{R}^{2}}\left|w_{n+1}\right|^{q-2} w_{n+1} \partial_{t} w_{n+1} e^{-\epsilon|x|} d x \\
&=- \int_{\mathbb{R}^{2}} \nabla \cdot\left(w_{n+1} B\left(u_{n}\right)\right)\left|w_{n+1}\right|^{q-2} w_{n+1} e^{-\epsilon|x|} d x \\
&-\int_{\mathbb{R}^{2}} \nabla \cdot\left(u_{n} B\left(w_{n}\right)\right)\left|w_{n+1}\right|^{q-2} w_{n+1} e^{-\epsilon|x|} d x \\
&-\nu \int_{\mathbb{R}^{2}}(-\Delta)^{\alpha / 2} w_{n+1}\left|w_{n+1}\right|^{q-2} w_{n+1} e^{-\epsilon|x|} d x \\
&= C_{1} \cdot\left(1-\frac{1}{q}\right) \int_{\mathbb{R}^{2}} u_{n}\left|w_{n+1}\right|^{q} e^{-\epsilon|x|} d x \\
&-\int_{\mathbb{R}^{2}} \nabla \cdot\left(u_{n} B\left(w_{n}\right)\right)\left|w_{n+1}\right|^{q-2} w_{n+1} e^{-\epsilon|x|} d x \\
&-\frac{\epsilon}{q} \int_{\mathbb{R}^{2}}\left(B\left(u_{n}\right) \cdot \frac{x}{|x|}\right)\left|w_{n+1}\right|^{q} e^{-\epsilon|x|} d x \\
&-\nu \int_{\mathbb{R}^{2}}(-\Delta)^{\alpha / 2} w_{n+1}\left|w_{n+1}\right|^{q-2} w_{n+1} e^{-\epsilon|x|} d x \\
& \lesssim\left.|| u_{n}\left\|_{L_{x}^{\infty}\left(\mathbb{R}^{2}\right)}+\right\| B\left(u_{n}\right)||_{L_{x}^{\infty}\left(\mathbb{R}^{2}\right)}\right) \int_{\mathbb{R}^{2}}\left|w_{n+1}\right|^{q} e^{-\epsilon|x|} d x \\
&-\nu \int_{\mathbb{R}^{2}}(-\Delta)^{\alpha / 2} w_{n+1}\left|w_{n+1}\right|^{q-2} w_{n+1} e^{-\epsilon|x|} d x \\
&-C_{1} \int_{\mathbb{R}^{2}} u_{n} w_{n}\left|w_{n+1}\right|^{q-2} w_{n+1} e^{-\epsilon|x|} d x \\
&-\int_{\mathbb{R}^{2}}\left(B\left(w_{n}\right) \cdot \nabla u_{n}\right)\left|w_{n+1}\right|^{q-2} w_{n+1} e^{-\epsilon|x|} d x \\
&= A_{1}+A_{2}+A_{3}+A_{4} \cdot
\end{aligned}
$$

We estimate each term separately.

Estimate of $A_{1}$. By the inequality

$$
\left\|B\left(u_{n}\right)\right\|_{L_{x}^{\infty}\left(\mathbb{R}^{2}\right)} \lesssim\left\|u_{n}\right\|_{L_{x}^{\infty}\left(\mathbb{R}^{2}\right)}+\left\|u_{n}\right\|_{L_{x}^{q}\left(\mathbb{R}^{2}\right)}
$$

and using the uniform estimate (3.30), we obtain

$$
\left|A_{1}\right| \lesssim \int_{\mathbb{R}^{2}}\left|w_{n+1}\right|^{q} e^{-\epsilon|x|} d x
$$

where the implicit constant depends only on $\left(\left\|u_{0}\right\|_{L_{x}^{q}\left(\mathbb{R}^{2}\right)}\right.$ and $\left.\left\|u_{0}\right\|_{H_{x}^{2}\left(\mathbb{R}^{2}\right)}\right)$. The same convention will be used below in the estimates of $A_{2}, A_{3}$ and $A_{4}$. 
Estimate of $A_{2}$. (Notice that in the inviscid case this term is 0 ). We can repeat the argument used in estimating (3.4) (see estimate (3.5)). Using (3.30), we then get

$$
\left|A_{2}\right| \lesssim\left\|w_{n+1}\right\|_{L_{x}^{\frac{q r}{r-1}}\left(\mathbb{R}^{2}\right)}^{r} \cdot \epsilon^{\alpha-\frac{2}{r}} \lesssim \epsilon^{\alpha-\frac{2}{r}},
$$

where $r$ is a positive number satisfying

$$
\frac{2}{\alpha}<r<\infty, \quad \text { if } 0<\alpha \leq 1
$$

and

$$
2 \leq r<\frac{2}{\alpha-1}, \quad \text { if } 1<\alpha<2 .
$$

Estimate of $A_{3}$. By Hölder's inequality and (3.30), we get

$$
\begin{aligned}
\left|A_{3}\right| & \lesssim\left\|u_{n}\right\|_{L_{x}^{\infty}\left(\mathbb{R}^{2}\right)} \cdot\left\|w_{n}\right\|_{L_{x}^{q}\left(\mathbb{R}^{2}\right)} \cdot\left(\int_{\mathbb{R}^{2}}\left|w_{n+1}\right|^{q} e^{-\epsilon \frac{q}{q-1}|x|} d x\right)^{\frac{q-1}{q}} \\
& \lesssim\left\|w_{n}\right\|_{L_{x}^{q}\left(\mathbb{R}^{2}\right)} \cdot\left(\int_{\mathbb{R}^{2}}\left|w_{n+1}\right|^{q} e^{-\epsilon|x|} d x\right)^{\frac{q-1}{q}}
\end{aligned}
$$

since $\frac{q}{q-1}>1$.

Estimate of $A_{4}$. Using Sobolev embedding, Hölder's inequality and (3.30), we have

$$
\begin{aligned}
\left\|\nabla u_{n} \cdot B\left(w_{n}\right)\right\|_{L_{x}^{q}\left(\mathbb{R}^{2}\right)} & \lesssim\left\|\nabla u_{n}\right\|_{L_{x}^{2}\left(\mathbb{R}^{2}\right)}\left\|(-\Delta)^{-1} \nabla w_{n}\right\|_{L_{x}^{\frac{2 q}{2-q}}\left(\mathbb{R}^{2}\right)} \\
& \lesssim\left\|u_{n}\right\|_{H_{x}^{2}\left(\mathbb{R}^{2}\right)}\left\|w_{n}\right\|_{L_{x}^{q}\left(\mathbb{R}^{2}\right)} \lesssim\left\|w_{n}\right\|_{L_{x}^{q}\left(\mathbb{R}^{2}\right)} .
\end{aligned}
$$

Hence Hölder gives us

$$
\left|A_{4}\right| \lesssim\left\|w_{n}\right\|_{L_{x}^{q}\left(\mathbb{R}^{2}\right)} \cdot\left(\int_{\mathbb{R}^{2}}\left|w_{n+1}\right|^{q} e^{-\epsilon|x|} d x\right)^{\frac{q-1}{q}} .
$$

Collecting the estimates (3.33), (3.34), (3.35) and (3.37), we finally obtain

$$
\begin{aligned}
& \frac{d}{d t} \int_{\mathbb{R}^{2}}\left|w_{n+1}(t)\right|^{q} e^{-\epsilon|x|} d x \lesssim \\
& \lesssim \int_{\mathbb{R}^{2}}\left|w_{n+1}(t)\right|^{q} e^{-\epsilon|x|} d x+\left\|w_{n}(t)\right\|_{L_{x}^{q}\left(\mathbb{R}^{2}\right)} \cdot\left(\int_{\mathbb{R}^{2}}\left|w_{n+1}(t)\right|^{q} e^{-\epsilon|x|} d x\right)^{\frac{q-1}{q}}+\epsilon^{\alpha-\frac{2}{r}} \\
& \lesssim \int_{\mathbb{R}^{2}}\left|w_{n+1}(t)\right|^{q} e^{-\epsilon|x|} d x+\left\|w_{n}(t)\right\|_{L_{x}^{q}\left(\mathbb{R}^{2}\right)}^{q}+\epsilon^{\alpha-\frac{2}{r}},
\end{aligned}
$$

where the last step follows from Young's inequality. Here the implicit constants depend only on $q,\left\|u_{0}\right\|_{H_{x}^{2}\left(\mathbb{R}^{2}\right)}$ and $\left\|u_{0}\right\|_{L_{x}^{q}\left(\mathbb{R}^{2}\right)}$. 
Now by choosing $T^{\prime}<T$ sufficiently small (but still depending only on $\left.\left\|u_{0}\right\|_{H_{x}^{2}\left(\mathbb{R}^{2}\right)},\left\|u_{0}\right\|_{L_{x}^{q}\left(\mathbb{R}^{2}\right)}\right)$ and using a Gronwall argument it is easy to see that (note that $w_{n}(0, x)=0$ )

$$
\sup _{0 \leq t \leq T^{\prime}} \int_{\mathbb{R}^{2}}\left|w_{n+1}(t, x)\right|^{q} e^{-\epsilon|x|} d x \leq \delta^{q} \sup _{0 \leq t \leq T^{\prime}}\left(\left\|w_{n}(t)\right\|_{L_{x}^{q}\left(\mathbb{R}^{2}\right)}^{q}+\epsilon^{\alpha-\frac{2}{r}}\right),
$$

where $0<\delta<1$ is a constant which is independent of $\epsilon$. Taking $\epsilon \rightarrow 0$ gives us

$$
\sup _{0 \leq t \leq T^{\prime}}\left\|w_{n+1}(t)\right\|_{L_{x}^{q}\left(\mathbb{R}^{2}\right)} \leq \delta \sup _{0 \leq t \leq T^{\prime}}\left\|w_{n}(t)\right\|_{L_{x}^{q}\left(\mathbb{R}^{2}\right)}
$$

This shows that $\left(u_{n}\right)_{n \geq 1}$ forms a Cauchy sequence in $C\left(\left[0, T^{\prime}\right], L_{x}^{q}\left(\mathbb{R}^{2}\right)\right)$. Therefore there exists a limiting function $u \in C\left(\left[0, T^{\prime}\right], L_{x}^{q}\left(\mathbb{R}^{2}\right)\right)$ such that $u_{n} \rightarrow u$ as $n \rightarrow \infty$.

Step 4. Properties of the limiting function $u$. By using the interpolation inequality:

$$
\|f\|_{H_{x}^{s^{\prime}}\left(\mathbb{R}^{2}\right)} \lesssim\|f\|_{L_{x}^{q}\left(\mathbb{R}^{2}\right)}^{\frac{s-s^{\prime}}{\frac{2}{q}-s}}\|f\|_{H_{x}^{s}\left(\mathbb{R}^{2}\right)}^{\frac{\frac{2}{q}-1+s^{\prime}}{\frac{2}{q}-1+s}}
$$

which holds for any $0 \leq s^{\prime} \leq s$ and $1 \leq q \leq 2$, and using (3.30), it is easy to see that $u_{n} \rightarrow u$ also in $C\left(\left[0, T^{\prime}\right], H_{x}^{s^{\prime}}\left(\mathbb{R}^{2}\right)\right)$ for any $s^{\prime}<s$. Therefore we have $u \in C\left(\left[0, T^{\prime}\right], H_{x}^{s^{\prime}}\left(\mathbb{R}^{2}\right) \cap L_{x}^{q}\left(\mathbb{R}^{2}\right)\right)$ for any $s^{\prime}<s$. By a standard argument $u$ is a classical solution to (1.1). We still have to show $u \in C\left(\left[0, T^{\prime}\right], H_{x}^{s}\left(\mathbb{R}^{2}\right)\right)$. It is clear that $u \in C_{w}\left(\left[0, T^{\prime}\right], H_{x}^{s}\left(\mathbb{R}^{2}\right)\right)$. By $(3.31)$, it is not difficult to show that

$$
\lim _{t \rightarrow 0}\|u(t)\|_{Y^{s}\left(\mathbb{R}^{2}\right)}=\left\|u_{0}\right\|_{Y^{s}\left(\mathbb{R}^{2}\right)}
$$

Together with the fact that $u \in C\left(\left[0, T^{\prime}\right], L_{x}^{q}\left(\mathbb{R}^{2}\right)\right)$, this implies

$$
\lim _{t \rightarrow 0}\|u(t)\|_{H_{x}^{s}\left(\mathbb{R}^{2}\right)}=\left\|u_{0}\right\|_{H_{x}^{s}\left(\mathbb{R}^{2}\right)},
$$

and therefore the strong continuity of $u$ at $t=0$ is proved (since $u \in$ $\left.C_{w}\left(\left[0, T^{\prime}\right], H_{x}^{s}\left(\mathbb{R}^{2}\right)\right)\right)$. To show the strong continuity at any $0<t \leq T^{\prime}$, we discuss two cases. In the inviscid case (i.e. $\nu=0$ ), then this case is rather simple and one can repeat the argument for $t=0$ to prove the strong continuity at $t$. In the case with diffusion (i.e. $\nu>0$ ), we use (3.30) to conclude that $u \in L_{t}^{2} H_{x}^{s+\frac{\alpha}{2}}\left(\left[0, T^{\prime}\right] \times \mathbb{R}^{2}\right)$. Consequently for any $\delta>0$ there exists $t-\delta<t_{0}<t$ such that $u\left(t_{0}\right) \in H_{x}^{s+\frac{\alpha}{2}}\left(\mathbb{R}^{2}\right)$. Taking $u\left(t_{0}\right)$ as initial data we obtain a solution in $C\left(\left[t_{0}, T^{\prime \prime}\right], H_{x}^{s^{\prime}}\left(\mathbb{R}^{2}\right)\right)$ for any $s^{\prime}<s+\frac{\alpha}{2}$. Here the time of existence $T^{\prime \prime}-t_{0}$ depends only on $\left(\left\|u\left(t_{0}\right)\right\|_{H_{x}^{2}\left(\mathbb{R}^{2}\right)}\right.$ and $\left.\left\|u\left(t_{0}\right)\right\|_{L_{x}^{q}\left(\mathbb{R}^{2}\right)}\right)$ and 
therefore has a uniform lower bound independent of $t_{0}$. By uniqueness of the solution and interpolation inequality (since $s+\frac{\alpha}{2}>s$ ) we obtain the strong continuity of $u$ at time $t$. Therefore we conclude $u \in C\left(\left[0, T^{\prime}\right], H_{x}^{s}\left(\mathbb{R}^{2}\right)\right)$. Finally we need to show that $u \in C^{1}\left([0, T), H_{x}^{s_{0}}\left(\mathbb{R}^{2}\right)\right)$ with $s_{0}=\min \{s-$ $\alpha, s-1\}$. This is rather straightforward. Let $0 \leq \tau_{0}, \tau<T^{\prime}$, by using (1.1) we then estimate

$$
\begin{aligned}
& \left\|\left(\partial_{t} u\right)(\tau)-\left(\partial_{t} u\right)\left(\tau_{0}\right)\right\|_{H_{x}^{s_{0}}\left(\mathbb{R}^{2}\right)} \leq \\
& \quad \leq\left\|\nabla \cdot\left(\left(u(\tau)-u\left(\tau_{0}\right)\right) \nabla K * u(\tau)\right)\right\|_{H_{x}^{s_{0}}\left(\mathbb{R}^{2}\right)} \\
& \quad+\left\|\nabla \cdot\left(u\left(\tau_{0}\right)\left(\nabla K *\left(u(\tau)-u\left(\tau_{0}\right)\right)\right)\right)\right\|_{H_{x}^{s_{0}}\left(\mathbb{R}^{2}\right)}+\nu\left\|(-\Delta)^{\alpha / 2}\left(u\left(\tau_{0}\right)-u(\tau)\right)\right\|_{H_{x}^{s_{0}\left(\mathbb{R}^{2}\right)}} \\
& \quad \leq\left\|\left(u(\tau)-u\left(\tau_{0}\right)\right) \nabla K * u(\tau)\right\|_{H_{x}^{s_{0}+1}\left(\mathbb{R}^{2}\right)} \\
& \quad+\left\|u\left(\tau_{0}\right)\left(\nabla K *\left(u(\tau)-u\left(\tau_{0}\right)\right)\right)\right\|_{H_{x}^{s_{0}+1}\left(\mathbb{R}^{2}\right)}+\nu\left\|u\left(\tau_{0}\right)-u(\tau)\right\|_{H_{x}^{s_{0}+\alpha}\left(\mathbb{R}^{2}\right)} .
\end{aligned}
$$

Using the fact that $H_{x}^{r}\left(\mathbb{R}^{2}\right)$ is an algebra when $r>1$, we have

$$
\begin{aligned}
& \left\|\left(\partial_{t} u\right)(\tau)-\left(\partial_{t} u\right)\left(\tau_{0}\right)\right\|_{H_{x}^{s_{0}}\left(\mathbb{R}^{2}\right)} \lesssim \\
& \quad \lesssim\left\|u(\tau)-u\left(\tau_{0}\right)\right\|_{H_{x}^{s_{0}+1}\left(\mathbb{R}^{2}\right)}\|\nabla K * u(\tau)\|_{H_{x}^{s_{0}+1}\left(\mathbb{R}^{2}\right)} \\
& \quad+\left\|u\left(\tau_{0}\right)\right\|_{H_{x}^{s_{0}+1}\left(\mathbb{R}^{2}\right)}\left\|\nabla K *\left(u(\tau)-u\left(\tau_{0}\right)\right)\right\|_{H_{x}^{s_{0}+1}\left(\mathbb{R}^{2}\right)}+\nu\left\|u(\tau)-u\left(\tau_{0}\right)\right\|_{H_{x}^{s_{0}+\alpha}\left(\mathbb{R}^{2}\right)} \\
& \quad \lesssim C\left\|u(\tau)-u\left(\tau_{0}\right)\right\|_{H_{x}^{s}\left(\mathbb{R}^{2}\right)} \rightarrow 0
\end{aligned}
$$

as we take $\tau \rightarrow \tau_{0}$. Therefore $\partial_{t} u \in C\left(\left[0, T^{\prime}\right), H_{x}^{s_{0}}\left(\mathbb{R}^{2}\right)\right)$ completing the proof of Theorem 1.1.

\section{Proof of Theorems 1.4 and 1.5}

Proof of Theorem 1.4. Let $u_{0} \in H_{x}^{s}\left(\mathbb{R}^{2}\right) \cap L_{x}^{q}\left(\mathbb{R}^{2}\right)$ with $s>3$ and $1<$ $q<2$. To prove the theorem it suffices to show the impossibility of the case when $u$ is the corresponding maximal lifespan solution in $C\left([0, T), L_{x}^{q}\left(\mathbb{R}^{2}\right) \cap\right.$ $\left.H_{x}^{s}\left(\mathbb{R}^{2}\right)\right)$ with $T<\infty$ and

$$
\int_{0}^{T}\|u(s)\|_{L_{x}^{\infty}\left(\mathbb{R}^{2}\right)} d s<\infty .
$$

We shall derive a contradiction from the above assumptions. By Theorem 1.1 we can continue the solution as long as we have a priori control of the $H_{x}^{2}\left(\mathbb{R}^{2}\right)+L_{x}^{q}\left(\mathbb{R}^{2}\right)$-norm. Since $\|u\|_{H_{x}^{2}\left(\mathbb{R}^{2}\right)} \lesssim\|u\|_{L_{x}^{q}\left(\mathbb{R}^{2}\right)}+\|u\|_{Y^{2}\left(\mathbb{R}^{2}\right)}($ see $(3.10))$, it suffices to control the $Y^{2}\left(\mathbb{R}^{2}\right)+L_{x}^{q}\left(\mathbb{R}^{2}\right)$-norm. By (3.6) (taking $v=u$ ), we have for any $q \leq p<\infty$

$$
\sup _{0 \leq t<T}\|u(t)\|_{L_{x}^{p}\left(\mathbb{R}^{2}\right)}<C_{1}<\infty,
$$

where $C_{1}$ is some constant depending on $u_{0}$ and $T$. 
Therefore we only need to control the $Y^{2}\left(\mathbb{R}^{2}\right)$ norm of solution. By (3.13) and using the fact that

$$
\left\|P_{>-20} u(t)\right\|_{\dot{H}_{x}^{2}\left(\mathbb{R}^{2}\right)} \lesssim\|u(t)\|_{Y^{2}\left(\mathbb{R}^{2}\right)}+\|u(t)\|_{L_{x}^{2}\left(\mathbb{R}^{2}\right)} \lesssim\|u(t)\|_{Y^{2}\left(\mathbb{R}^{2}\right)}+C_{1},
$$

we have for any $0<t<T$,

$$
\frac{d}{d t}\|u(t)\|_{Y^{2}\left(\mathbb{R}^{2}\right)}^{2} \lesssim\left(\|u(t)\|_{L_{x}^{\infty}\left(\mathbb{R}^{2}\right)}+\|D B(u)\|_{L_{x}^{\infty}\left(\mathbb{R}^{2}\right)}\right)\left(\|u(t)\|_{Y^{2}\left(\mathbb{R}^{2}\right)}^{2}+C_{1}^{2}\right) .
$$

By Lemma 2.6 and (4.1), we have

$$
\begin{aligned}
\|D B(u(t))\|_{L_{x}^{\infty}\left(\mathbb{R}^{2}\right)} & \lesssim \log \left(e+\|u(t)\|_{\dot{H}_{x}^{2}\left(\mathbb{R}^{2}\right)}\right)\left(1+\|u(t)\|_{L_{x}^{\infty}\left(\mathbb{R}^{2}\right)}\right)+\|u(t)\|_{L_{x}^{2}\left(\mathbb{R}^{2}\right)} \\
& \lesssim \log \left(C_{2}+\|u(t)\|_{Y^{2}\left(\mathbb{R}^{2}\right)}\right)\left(1+\|u(t)\|_{L_{x}^{\infty}\left(\mathbb{R}^{2}\right)}\right)+C_{3},
\end{aligned}
$$

where $C_{2}>e, C_{3}>0$ are constants, and we have used the fact that

$$
\|u(t)\|_{\dot{H}_{x}^{2}\left(\mathbb{R}^{2}\right)} \lesssim\|u(t)\|_{Y^{2}\left(\mathbb{R}^{2}\right)}+\|u(t)\|_{L_{x}^{2}\left(\mathbb{R}^{2}\right)} \lesssim\|u(t)\|_{Y^{2}\left(\mathbb{R}^{2}\right)}+C_{1} .
$$

Thus we obtain the following inequality

$$
\begin{aligned}
\frac{d}{d t}\left(C_{4}+\right. & \left.\|u(t)\|_{Y^{2}\left(\mathbb{R}^{2}\right)}^{2}\right) \leq \\
& \leq C_{5}\left(1+\|u(t)\|_{L_{x}^{\infty}\left(\mathbb{R}^{2}\right)}\right)\left(C_{4}+\|u(t)\|_{Y^{2}\left(\mathbb{R}^{2}\right)}^{2}\right) \log \left(C_{4}+\|u(t)\|_{Y^{2}\left(\mathbb{R}^{2}\right)}^{2}\right),
\end{aligned}
$$

where $C_{4}>e, C_{5}>0$ are constants. Now a Gronwall argument shows that for any $0<t<T$,

$$
\|u(t)\|_{Y^{2}\left(\mathbb{R}^{2}\right)}^{2} \leq \exp \left(\exp \left(C_{5} \int_{0}^{t}\left(1+\|u(s)\|_{L_{x}^{\infty}\left(\mathbb{R}^{2}\right)}\right) d s\right)\right)\left(C_{4}+\left\|u_{0}\right\|_{Y^{2}\left(\mathbb{R}^{2}\right)}^{2}\right) .
$$

This shows that $\|u(t)\|_{Y^{2}\left(\mathbb{R}^{2}\right)}$ can be controlled up to time $T$ and therefore we have obtained a contradiction. The Theorem is proved.

Proof of Theorem 1.5. We divide the proof into two steps.

Step 1. Nonnegativity of the solution. Let $u_{0} \in H_{x}^{s}\left(\mathbb{R}^{2}\right) \cap L_{x}^{q}\left(\mathbb{R}^{2}\right)$ for some $s>3$ and $1<q<2$. Assume $u_{0} \geq 0$ and let $u$ be the corresponding maximal lifespan solution obtained in Theorem 1.1. Let $0<T^{\prime}<T$ and denote $\Omega_{T^{\prime}}=\mathbb{R}^{2} \times\left(0, T^{\prime}\right]$. By Theorem 1.1 we have $u \in C\left(\left[0, T^{\prime}\right], H_{x}^{s}\left(\mathbb{R}^{2}\right) \cap\right.$ $\left.L_{x}^{q}\left(\mathbb{R}^{2}\right)\right) \cap C^{1}\left(\left[0, T^{\prime}\right], H_{x}^{s}\left(\mathbb{R}^{2}\right)\right)$ with $s_{0}=\min \{s-\alpha, s-1\}>1$. By Sobolev embedding we have

$$
u \in C_{t, x}^{1,2}\left(\Omega_{T^{\prime}}\right) \cap C_{t, x}^{0}\left(\bar{\Omega}_{T^{\prime}}\right) \cap L_{t, x}^{2}\left(\Omega_{T^{\prime}}\right) .
$$

It is not difficult to check that $u_{0} \in C^{2}\left(\mathbb{R}^{2}\right), f=\nabla K * u \in C_{t, x}^{0,1}\left(\Omega_{T^{\prime}}\right)$ with

$$
\sup _{\Omega_{T^{\prime}}}|\operatorname{div}(f)|=\sup _{\Omega_{T^{\prime}}}|\Delta K * u| \lesssim\|u\|_{L_{t}^{\infty} H_{x}^{s}\left(\Omega_{T^{\prime}}\right)}<\infty .
$$


Also we have

$$
\sup _{\Omega_{T^{\prime}}}\left(\left|\partial_{t} u\right|+|D u|+\left|D^{2} u\right|\right)+\sup _{\bar{\Omega}_{T^{\prime}}}|u| \lesssim\|u\|_{L_{t}^{\infty} H_{x}^{s}\left(\Omega_{T^{\prime}}\right)}<\infty .
$$

Therefore by Lemma 2.7, we conclude $u \geq 0$ in $\Omega_{T^{\prime}}$. Since $T^{\prime}$ is arbitrary we conclude $u(t) \geq 0$ for any $0 \leq t<T$.

Step 2. $L_{x}^{1}\left(\mathbb{R}^{2}\right)$ conservation of the solution. Assume that $u_{0} \in H_{x}^{s}\left(\mathbb{R}^{2}\right) \cap$ $L_{x}^{1}\left(\mathbb{R}^{2}\right)$ with $s>3$ and $u_{0} \geq 0$. This easily implies that $u_{0} \in H_{x}^{s}\left(\mathbb{R}^{2}\right) \cap L_{x}^{q}\left(\mathbb{R}^{2}\right)$ for any $1<q<\infty$. Let $u$ be the corresponding maximal life span solution obtained in Theorem 1.1. Then clearly $u \in C\left([0, T), H_{x}^{s}\left(\mathbb{R}^{2}\right) \cap L_{x}^{q}\left(\mathbb{R}^{2}\right)\right)$ for any $1<q<2$. By the result of step 1 we obtain $u(t) \geq 0$ for any $0 \leq t<T$. To prove $L_{x}^{1}$ conservation, it is enough to prove the statement on any interval $\left[0, T^{\prime}\right]$ for $T^{\prime}<T$. By the fact that $u \in C\left(\left[0, T^{\prime}\right], L_{x}^{q}\left(\mathbb{R}^{2}\right)\right)$, we have

$$
\sup _{0 \leq t \leq T^{\prime}}\|u(t)\|_{L_{x}^{q}\left(\mathbb{R}^{2}\right)} \leq C_{1}<\infty
$$

where $C_{1}$ is a positive constant. Now take $\psi \in C_{c}^{\infty}\left(\mathbb{R}^{2}\right)$ such that $\psi(x) \equiv 1$ for $|x| \leq 1$ and $\psi \geq 0$. Take $R>0$, we then compute by using (1.1),

$$
\begin{aligned}
\left|\frac{d}{d t} \int_{\mathbb{R}^{2}} u(t, x) \psi\left(\frac{x}{R}\right) d x\right|= & \left|-\int_{\mathbb{R}^{2}} \nabla \cdot(u B(u)) \psi\left(\frac{x}{R}\right) d x-\nu \int_{\mathbb{R}^{2}}(-\Delta)^{\alpha / 2} u(t, x) \psi\left(\frac{x}{R}\right) d x\right| \\
= & \left|\frac{1}{R} \int_{\mathbb{R}^{2}} u B(u) \cdot(\nabla \psi)\left(\frac{x}{R}\right) d x-\nu \frac{1}{R^{\alpha}} \int_{\mathbb{R}^{2}} u(t, x)\left((-\Delta)^{\alpha / 2} \psi\right)\left(\frac{x}{R}\right) d x\right| \\
\leq & \frac{1}{R} \cdot R^{\frac{2(q-1)}{q}}\|u(t)\|_{L_{x}^{q}\left(\mathbb{R}^{2}\right)}\|\nabla \psi\|_{L_{x}^{\frac{q}{q-1}}\left(\mathbb{R}^{2}\right)}\|B(u)\|_{L_{x}^{\infty}\left(\mathbb{R}^{2}\right)} \\
& \quad+\nu \frac{1}{R^{\alpha}} \cdot R^{\frac{2(q-1)}{q}}\|u(t)\|_{L_{x}^{q}\left(\mathbb{R}^{2}\right)}\left\|(-\Delta)^{\alpha / 2} \psi\right\|_{L_{x}^{\frac{q}{q-1}}\left(\mathbb{R}^{2}\right)},
\end{aligned}
$$

where we will take $q=1+\epsilon$ with $\epsilon>0$ sufficiently small. Since $u \in$ $C\left(\left[0, T^{\prime}\right], H_{x}^{s}\left(\mathbb{R}^{2}\right) \cap L_{x}^{q}\left(\mathbb{R}^{2}\right)\right)$,

$$
\|B(u)\|_{L_{x}^{\infty}\left(\mathbb{R}^{2}\right)} \leq C\left\||\nabla|^{-2} \nabla u\right\|_{L_{x}^{\infty}\left(\mathbb{R}^{2}\right)} \lesssim\left(\|u\|_{L_{x}^{q}\left(\mathbb{R}^{2}\right)}+\|u\|_{H_{x}^{s}\left(\mathbb{R}^{2}\right)}\right) \lesssim C_{2},
$$

where $C_{2}$ is a constant. By (4.2) (4.3) and taking $\epsilon$ sufficiently small, we have for any $0<t \leq T^{\prime}$,

$$
\left|\frac{d}{d t} \int_{\mathbb{R}^{2}} u(t, x) \psi\left(\frac{x}{R}\right) d x\right| \leq \frac{C_{3}}{R^{\frac{1}{2}}}+\frac{C_{4}}{R^{\frac{\alpha}{2}}},
$$

where $C_{3}, C_{4}$ are constants. 
Using (4.4) and integrating over the time interval $[0, t]$ with $0<t \leq T$, we obtain

$$
\int_{|x| \leq R} u(t, x) d x \leq\left\|u_{0}\right\|_{L_{x}^{1}\left(\mathbb{R}^{2}\right)}+T\left(\frac{C_{3}}{R^{\frac{1}{2}}}+\frac{C_{4}}{R^{\frac{\alpha}{2}}}\right),
$$

where we have used the fact that $\psi \geq 0$ and $\psi(x) \equiv 1$ for $|x| \leq 1$. Since $u$ is nonnegative, sending $R \rightarrow \infty$ and using Lebesgue's Monotone Convergence Theorem we immediately obtain

$$
\|u(t)\|_{L_{x}^{1}\left(\mathbb{R}^{2}\right)} \leq\left\|u_{0}\right\|_{L_{x}^{1}\left(\mathbb{R}^{2}\right)} .
$$

On the other hand, by using (4.4) again and integrating over $[0, t]$ gives us

$$
\|u(t)\|_{L_{x}^{1}\left(\mathbb{R}^{2}\right)} \geq \int_{\mathbb{R}^{2}} u(t, x) \psi\left(\frac{x}{R}\right) d x \geq \int_{\mathbb{R}^{2}} u_{0}(x) \psi\left(\frac{x}{R}\right) d x-T\left(\frac{C_{3}}{\sqrt{R}}+\frac{C_{4}}{R^{\frac{\alpha}{2}}}\right),
$$

sending $R \rightarrow \infty$ gives us $\|u(t)\|_{L_{x}^{1}\left(\mathbb{R}^{2}\right)} \geq\left\|u_{0}\right\|_{L_{x}^{1}\left(\mathbb{R}^{2}\right)}$ and therefore the $L_{x}^{1}$ conservation is proved. The theorem is proved.

\section{Proof of Theorem 1.7}

Proof of Theorem 1.7. Assume $u_{0} \in H_{x}^{s}\left(\mathbb{R}^{2}\right) \cap L_{x}^{q}\left(\mathbb{R}^{2}\right)$ with $s>3$, $1 \leq q<2$ and $u_{0} \geq 0$. Let $u$ be the corresponding maximal-lifespan solution. By Theorem 1.4, to prove that $u$ is global it suffices for us to control the $L_{x}^{\infty}$-norm of $u$. By Theorem 1.5 we have $u(t) \geq 0$ for any $t \geq 0$. Now let $1<p<\infty$ be arbitrary, by a similar calculation as in the a priori estimate in Section 3 (see equation (3.2)), we have

$$
\begin{aligned}
\frac{1}{p} \frac{d}{d t} \int_{\mathbb{R}^{2}}|u|^{p} e^{-\epsilon|x|} d x= & \\
= & -C_{1} \cdot\left(1-\frac{1}{p}\right) \int_{\mathbb{R}^{2}}|u|^{p+1} e^{-\epsilon|x|} d x-\frac{\epsilon}{p} \int_{\mathbb{R}^{2}}\left(B(u) \cdot \frac{x}{|x|}\right)|u|^{p} e^{-\epsilon|x|} d x \\
& \quad-\nu \int_{\mathbb{R}^{2}}(-\Delta)^{\alpha / 2} u|u|^{p-2} u e^{-\epsilon|x|} d x \\
1) & \frac{\epsilon}{p}\|B(u)\|_{L_{x}^{\infty}\left(\mathbb{R}^{2}\right)}\|u\|_{L_{x}^{p}\left(\mathbb{R}^{2}\right)}^{p}+\frac{C}{p} \nu t \epsilon^{\alpha-\frac{2}{r}}\|u\|_{L_{x}^{p-p}}^{p}\left(\mathbb{R}^{2}\right)
\end{aligned}
$$

where the last inequality follows from (3.5). Let $0 \leq t_{1}<t_{2}$ be arbitrary but in the lifespan of $u$. By Theorem 1.4 we have $u \in C\left(\left[0, t_{2}\right], L_{x}^{q}\left(\mathbb{R}^{2}\right) \cap H_{x}^{s}\left(\mathbb{R}^{2}\right)\right)$, and this gives

$$
\begin{aligned}
\sup _{0 \leq t \leq t_{2}}\left(\|B(u)(t)\|_{L_{x}^{\infty}\left(\mathbb{R}^{2}\right)} \cdot\|u(t)\|_{L_{x}^{p}\left(\mathbb{R}^{2}\right)}^{p}\right. & \left.+C \cdot\|u(t)\|_{L_{x}^{p r}}^{p}{ }^{\frac{p r}{p-1}}\right) \leq \\
& \leq C\left(\left\|u_{0}\right\|_{L_{x}^{q}\left(\mathbb{R}^{2}\right)},\left\|u_{0}\right\|_{H_{x}^{s}\left(\mathbb{R}^{2}\right)}, t_{2}\right)<\infty .
\end{aligned}
$$


Plugging this estimate into (5.1) and integrating on the time interval $\left[t_{1}, t_{2}\right]$, and we get

$$
\frac{1}{p} \int_{\mathbb{R}^{2}}\left|u\left(t_{2}, x\right)\right|^{p} e^{-\epsilon|x|} d x \leq \frac{1}{p} \int_{\mathbb{R}^{2}}\left|u\left(t_{1}, x\right)\right|^{p} e^{-\epsilon|x|} d x+O\left(\epsilon+\epsilon^{\alpha-\frac{2}{r}}\right),
$$

where the term $O\left(\epsilon+\epsilon^{\alpha-\frac{2}{r}}\right) \rightarrow 0$ as $\epsilon \rightarrow 0$. Taking $\epsilon \rightarrow 0$ in the above expression, we immediately obtain

$$
\left\|u\left(t_{2}\right)\right\|_{L_{x}^{p}\left(\mathbb{R}^{2}\right)} \leq\left\|u\left(t_{1}\right)\right\|_{L_{x}^{p}\left(\mathbb{R}^{2}\right)},
$$

for any $1<p<\infty$. By Sobolev embedding we know $\|u(t)\|_{L_{x}^{\infty}\left(\mathbb{R}^{2}\right)}<\infty$ for any $0 \leq t \leq t_{2}$. Taking $p \rightarrow \infty$ in (5.2), we finally get

$$
\left\|u\left(t_{2}\right)\right\|_{L_{x}^{\infty}\left(\mathbb{R}^{2}\right)} \leq\left\|u\left(t_{1}\right)\right\|_{L_{x}^{\infty}\left(\mathbb{R}^{2}\right)}, \quad \forall t_{1}<t_{2} .
$$

This estimate shows that $\|u(t)\|_{L_{x}^{\infty}\left(\mathbb{R}^{2}\right)}$ is under control for any $t$ and therefore our solution is global. The same estimate also shows that $\|u(t)\|_{L_{x}^{r}\left(\mathbb{R}^{2}\right)}, 1<$ $r \leq \infty$ is a non-increasing function of $t$. The rest of the statements of Theorem 1.7 except the estimate (1.4) now follows easily from Theorem 1.5. Therefore it remains for us to prove the estimate (1.4). For this we will use the method of characteristics. Define the characteristic lines $X(t, \alpha)$ which solve the following ODE:

$$
\left\{\begin{array}{l}
\frac{d}{d t} X(t, \alpha)=B(u(t))(X(t, \alpha)) \\
X(0, \alpha)=\alpha, \quad \alpha \in \mathbb{R}^{2}
\end{array}\right.
$$

Since our solution $u \in C\left([0, \infty), L_{x}^{q}\left(\mathbb{R}^{2}\right) \cap H_{x}^{s}\left(\mathbb{R}^{2}\right)\right)$, it is rather easy to check that $X(t, \alpha)$ are well defined for all time. Denote $\tilde{u}(t, \alpha)=u(t, X(t, \alpha))$. Then by the definition of the characteristics, we have

$$
\frac{d}{d t} \tilde{u}(t, \alpha)=-C_{1} \tilde{u}(t, \alpha)^{2}
$$

where $C_{1}$ is the same constant as in (1.3). It is rather straightforward to solve the ODE (5.3) and this gives us

$$
\tilde{u}(t, \alpha)=\frac{u_{0}(\alpha)}{C_{1} u_{0}(\alpha) t+1} .
$$

We then have

$$
\|u(t)\|_{L_{x}^{\infty}\left(\mathbb{R}^{2}\right)} \leq \sup _{\alpha \in \mathbb{R}^{2}} \tilde{u}(t, \alpha) \leq \frac{\left\|u_{0}\right\|_{L_{x}^{\infty}\left(\mathbb{R}^{2}\right)}}{1+C_{1}\left\|u_{0}\right\|_{L_{x}^{\infty}\left(\mathbb{R}^{2}\right)} t} .
$$


Interpolating this $L^{\infty}$ bound with the trivial $L_{x}^{1}$ bound, we get for any $1 \leq p \leq \infty$,

$$
\begin{aligned}
\|u(t)\|_{L_{x}^{p}\left(\mathbb{R}^{2}\right)} & \leq\left\|u_{0}\right\|_{L_{x}^{1}\left(\mathbb{R}^{2}\right)}^{\frac{1}{p}}\|u(t)\|_{L_{x}^{\infty}\left(\mathbb{R}^{2}\right)}^{1-\frac{1}{p}} \\
& \leq\left\|u_{0}\right\|_{L_{x}^{1}\left(\mathbb{R}^{2}\right)}^{\frac{1}{p}} \cdot\left\|u_{0}\right\|_{L_{x}^{\infty}\left(\mathbb{R}^{2}\right)}^{1-\frac{1}{p}} \cdot\left(1+C_{1}\left\|u_{0}\right\|_{L_{x}^{\infty}\left(\mathbb{R}^{2}\right)} t\right)^{-\left(1-\frac{1}{p}\right)} .
\end{aligned}
$$

This finishes the proof of the upper bound in Theorem 1.7, equation (1.4). For the lower bound we compute directly the $L_{x}^{p}$-norm of $u$ for $1<p<\infty$ as

$$
\begin{aligned}
\frac{1}{p} \frac{d}{d t}\|u(t)\|_{L_{x}^{p}\left(\mathbb{R}^{2}\right)}^{p} & =-C_{1} \cdot\left(1-\frac{1}{p}\right)\|u(t)\|_{L_{x}^{p+1}\left(\mathbb{R}^{2}\right)}^{p+1} \\
& \geq-C_{1} \cdot\left(1-\frac{1}{p}\right)\|u(t)\|_{L_{x}^{\infty}\left(\mathbb{R}^{2}\right)}\|u(t)\|_{L_{x}^{p}\left(\mathbb{R}^{2}\right)}^{p}
\end{aligned}
$$

Integrating in the time and using the upper bound of $\|u(t)\|_{L_{x}^{\infty}\left(\mathbb{R}^{2}\right)}$ in (5.5), we get

$$
\begin{aligned}
\|u(t)\|_{L_{x}^{p}\left(\mathbb{R}^{2}\right)} & \geq\left\|u_{0}\right\|_{L_{x}^{p}\left(\mathbb{R}^{2}\right)} \cdot \exp \left(-C_{1}\left(1-\frac{1}{p}\right) \int_{0}^{t}\|u(\tau)\|_{L_{x}^{\infty}\left(\mathbb{R}^{2}\right)} d \tau\right) \\
& \geq\left\|u_{0}\right\|_{L_{x}^{p}\left(\mathbb{R}^{2}\right)} \exp \left(-C_{1} \cdot\left(1-\frac{1}{p}\right) \int_{0}^{t} \frac{\left\|u_{0}\right\|_{L_{x}^{\infty}\left(\mathbb{R}^{2}\right)}}{1+C_{1}\left\|u_{0}\right\|_{L_{x}^{\infty}\left(\mathbb{R}^{2}\right)}} d \tau\right) \\
& \geq\left\|u_{0}\right\|_{L_{x}^{p}\left(\mathbb{R}^{2}\right)}\left(1+C_{1}\left\|u_{0}\right\|_{L_{x}^{\infty}\left(\mathbb{R}^{2}\right)} t\right)^{-\left(1-\frac{1}{p}\right)} .
\end{aligned}
$$

This gives the lower estimate for $1<p<\infty$. The corresponding estimate for $p=1$ is trivial. For $p=\infty$, just observe that the inequality in (5.5) is actually an equality. The theorem is proved.

Remark 5.1. We again point out that the role of the function $e^{-\epsilon|x|}$ used in the proof of Theorem 1.7 is to regularize the integral involving the fractional diffusion in the case $1<p<2$. In fact if $2 \leq p<\infty$, we do not need to use this regularization and directly compute by using Lemma 2.8,

$$
\begin{aligned}
\frac{1}{p} \frac{d}{d t} \int_{\mathbb{R}^{2}}|u|^{p} d x & =-C_{1} \cdot\left(1-\frac{1}{p}\right) \int_{\mathbb{R}^{2}}|u|^{p+1} d x-\nu \int_{\mathbb{R}^{2}}(-\Delta)^{\alpha / 2} u|u|^{p-2} u d x \\
& \leq-C_{1} \cdot\left(1-\frac{1}{p}\right) \int_{\mathbb{R}^{2}}|u|^{p+1} d x \leq 0 .
\end{aligned}
$$

Note that in the case $2 \leq p<\infty$ the integral involving the fractional diffusion term converges since

$$
\begin{aligned}
\left\|(-\Delta)^{\alpha / 2} u u^{p-1}\right\|_{L_{x}^{1}\left(\mathbb{R}^{2}\right)} & \lesssim\left\|(-\Delta)^{\alpha / 2} u\right\|_{L_{x}^{p}\left(\mathbb{R}^{2}\right)}\|u\|_{L_{x}^{p}\left(\mathbb{R}^{2}\right)}^{p-1} \\
& \lesssim C\left(\|u\|_{L_{x}^{q}\left(\mathbb{R}^{2}\right)},\|u\|_{H_{x}^{s}\left(\mathbb{R}^{2}\right)}\right)<\infty .
\end{aligned}
$$




\section{Proof of Theorem 1.9}

Before we present the proof of Theorem 1.9, we establish a slightly weaker proposition which uses a virial argument. The actual proof of Theorem 1.9 uses a different argument. The proof of Proposition 6.1 can be compared with the proof of Theorem 1.10 where a truncated virial argument is used (see Section 7).

Proposition 6.1. (Blow up in the attractive case with no diffusion: virial argument). Assume $\mu=-1$ and $\nu=0$. Let $u_{0} \in H_{x}^{s}\left(\mathbb{R}^{2}\right) \cap L_{x}^{1}\left(\mathbb{R}^{2}\right)$ with $s>3$ and $u_{0} \geq 0$, with $u_{0}$ not identically 0 . Assume further that $|x|^{2} u_{0} \in L_{x}^{1}\left(\mathbb{R}^{2}\right)$, then the corresponding solution $u$ blows up in finite time, i.e., there exists $T<\infty$ such that

$$
\lim _{t \rightarrow T}\|u(t)\|_{L_{x}^{\infty}\left(\mathbb{R}^{2}\right)}=+\infty .
$$

In particular, any solution with smooth compactly supported initial data (nonnegative and with nonzero mass) will blow up in finite time. Moreover on the time interval $[0, T)$, for any $1 \leq r \leq \infty$, the norm $\|u(t)\|_{L_{x}^{r}\left(\mathbb{R}^{2}\right)}$ is a non-decreasing function of $t$.

Proof of Proposition 6.1. Let $u_{0} \in H_{x}^{s}\left(\mathbb{R}^{2}\right) \cap L_{x}^{1}\left(\mathbb{R}^{2}\right)$ with $s>3$. Assume $u_{0} \geq 0$ and $|x|^{2} u_{0} \in L_{x}^{1}\left(\mathbb{R}^{2}\right)$. Let $u$ be the corresponding maximal lifespan solution obtained by Theorem 1.1. By Theorem 1.4, to prove the finite time blowup of $L_{x}^{\infty}$-norm of $u$, we only need to show that $u$ cannot be a global solution. Indeed assume this is the case. We first prove that for any $t>0$, $|x|^{2} u(t, \cdot) \in L_{x}^{1}\left(\mathbb{R}^{d}\right)$. Let $0<\epsilon<1$ and $T>0$ be fixed but arbitrary. We then compute for $0<t \leq T$,

$$
\begin{aligned}
& \frac{d}{d t} \int_{\mathbb{R}^{2}} u(t, x)|x|^{2} e^{-\epsilon|x|} d x=-\int_{\mathbb{R}^{2}} \nabla \cdot(u B(u))|x|^{2} e^{-\epsilon|x|} d x \\
& \quad=\int_{\mathbb{R}^{2}} u B(u) \cdot 2 x e^{-\epsilon|x|} d x-\epsilon \int_{\mathbb{R}^{2}} u B(u) \cdot x|x| e^{-\epsilon|x|} d x \\
& \quad \leq \int_{\mathbb{R}^{2}} u\left(|B(u)|^{2}+|x|^{2}\right) e^{-\epsilon|x|} d x+\|B(u)\|_{L_{x}^{\infty}\left(\mathbb{R}^{2}\right)} \int_{\mathbb{R}^{2}} u(t, x)|x|^{2} e^{-\epsilon|x|} d x \\
& \quad \leq\|B(u)\|_{L_{x}^{\infty}\left(\mathbb{R}^{2}\right)}^{2}\left\|u_{0}\right\|_{L_{x}^{1}\left(\mathbb{R}^{2}\right)}+\left(1+\|B(u)\|_{L_{x}^{\infty}\left(\mathbb{R}^{2}\right)}\right) \int_{\mathbb{R}^{2}} u(t, x)|x|^{2} e^{-\epsilon|x|} d x
\end{aligned}
$$

where the last step follows from the $L_{x}^{1}$ preservation of $u$. Now we have

$$
\begin{aligned}
\|B(u)\|_{L_{x}^{\infty}\left(\mathbb{R}^{2}\right)} & \lesssim\left\|(-\Delta)^{-1} \nabla u\right\|_{L_{x}^{\infty}\left(\mathbb{R}^{2}\right)} \lesssim\|u\|_{L_{x}^{\infty}\left(\mathbb{R}^{2}\right)}+\|u\|_{L_{x}^{1}\left(\mathbb{R}^{2}\right)} \\
& \leq C\left(T,\left\|u_{0}\right\|_{L_{x}^{1}\left(\mathbb{R}^{2}\right)},\left\|u_{0}\right\|_{H_{x}^{s}\left(\mathbb{R}^{2}\right)}\right)<\infty .
\end{aligned}
$$


A Gronwall argument then yields that

$$
\sup _{0 \leq t \leq T} \int_{\mathbb{R}^{2}} u(t, x)|x|^{2} e^{-\epsilon|x|} d x \leq C\left(T,\left\|u_{0}\right\|_{L_{x}^{1}\left(\mathbb{R}^{2}\right)},\left\|u_{0}\right\|_{H_{x}^{s}\left(\mathbb{R}^{2}\right)}\right)<\infty .
$$

Letting $\epsilon \rightarrow 0$ and using Lebesgue's Monotone Convergence Theorem immediately we conclude that $|x|^{2} u(t, x) \in L_{x}^{1}\left(\mathbb{R}^{2}\right)$. To prove finite time blowup, we compute by using (1.2),

$$
\begin{aligned}
\frac{d}{d t} \int_{\mathbb{R}^{2}} u(t, x)|x|^{2} d x=-\int_{\mathbb{R}^{2}} \nabla \cdot(u B(u))|x|^{2} d x \\
\quad=-2 \int_{\mathbb{R}_{x}^{2}} \int_{\mathbb{R}_{y}^{2}} x \cdot\left(\frac{x-y}{|x-y|^{2}}\right) u(x) u(y) d x d y=-\left\|u_{0}\right\|_{L_{x}^{1}\left(\mathbb{R}^{2}\right)}^{2},
\end{aligned}
$$

where the last step follows from symmetrizing the integral in $x$ and $y$. It is now clear that the integral $\int_{\mathbb{R}^{2}} u(t, x)|x|^{2} d x$ must become negative in finite time. This is a contradiction and thus we have proved the finite time blowup. Lastly to show the monotonicity of $\|u(t)\|_{L_{x}^{r}\left(\mathbb{R}^{2}\right)}, 1<r \leq \infty$, before the blowup time $T$, we calculate for any $1<p<\infty$,

$\frac{1}{p} \frac{d}{d t} \int_{\mathbb{R}^{2}}|u|^{p} d x=\left(1-\frac{1}{p}\right) \int_{\mathbb{R}^{2}}(\nabla \cdot B(u))|u|^{p} d x=\left(1-\frac{1}{p}\right) \int_{\mathbb{R}^{2}} C_{1} u^{p+1} d x \geq 0$.

Therefore we conclude that for any $0 \leq t_{1}<t_{2}<T,\left\|u\left(t_{1}\right)\right\|_{L_{x}^{p}\left(\mathbb{R}^{2}\right)} \leq$ $\left\|u\left(t_{2}\right)\right\|_{L_{x}^{p}\left(\mathbb{R}^{2}\right)}$. Taking $p \rightarrow \infty$ quickly yields the result.

Proof of Theorem 1.9. Instead of using the virial argument, we will use the method of characteristics which yields slightly better results. Define the characteristic lines $X(t, \alpha)$ which solve the following ODE:

$$
\left\{\begin{array}{l}
\frac{d}{d t} X(t, \alpha)=B(u(t))(X(t, \alpha)), \\
X(0, \alpha)=\alpha, \quad \alpha \in \mathbb{R}^{2} .
\end{array}\right.
$$

It is not difficult to check that $X(t, \alpha)$ are well defined on a time interval $\left[0, T^{\prime}\right)$ as long as the corresponding solution $u \in C\left(\left[0, T^{\prime}\right), L_{x}^{q}\left(\mathbb{R}^{2}\right) \cap H_{x}^{s}\left(\mathbb{R}^{2}\right)\right)$. By a slight abuse of notation we denote $u(t, \alpha)=u(t, X(t, \alpha))$. Then by the definition of the characteristics, we have

$$
\frac{d}{d t} u(t, \alpha)=C_{1} u(t, \alpha)^{2},
$$

where $C_{1}$ is the same constant as in (1.3). It is rather straightforward to solve the ODE (6.1) and this gives us

$$
u(t, \alpha)=\frac{u_{0}(\alpha)}{1-C_{1} u_{0}(\alpha) t} .
$$


Now define

$$
M=\sup _{\alpha \in \mathbb{R}^{2}} u_{0}(\alpha)
$$

Since by assumption $u_{0} \in L_{x}^{q}\left(\mathbb{R}^{2}\right) \cap H_{x}^{s}\left(\mathbb{R}^{2}\right)$ and the set $\left\{y: u_{0}(y)>0\right\}$ is nonempty, we conclude that $0<M<\infty$ and there exists $\alpha^{*} \in \mathbb{R}^{2}$ such that $u_{0}\left(\alpha^{*}\right)=M$. Let $T=\frac{1}{C_{1} M}$. We first show that the maximal lifespan of $u$ is given by $[0, T)$. Assume the lifespan of $u$ is given by $\left[0, T^{\prime}\right)$. Clearly $T^{\prime} \leq T$ since

$$
\limsup _{t \rightarrow T}\|u(t)\|_{L_{x}^{\infty}\left(\mathbb{R}^{2}\right)} \geq \lim _{t \rightarrow T} u\left(t, \alpha^{*}\right)=\infty,
$$

where the last equality follows from (6.2). On the other hand if $T^{\prime}<T$, then by (6.2) we have

$$
\limsup _{t \rightarrow T^{\prime}}\|u(t)\|_{L_{x}^{\infty}\left(\mathbb{R}^{2}\right)} \leq \limsup _{t \rightarrow T^{\prime}}\left(\sup _{\alpha \in \mathbb{R}^{2}}\left|\frac{u_{0}(\alpha)}{1-C_{1} u_{0}(\alpha) t}\right|\right) \leq \frac{\left\|u_{0}\right\|_{L_{x}^{\infty}\left(\mathbb{R}^{2}\right)}}{1-\frac{T^{\prime}}{T}}<\infty .
$$

This is obviously a contradiction to the blowup criteria and we conclude that the maximal lifespan of $u$ is precisely given by $[0, T)$. It remains for us to verify the blowup rate (1.5). The lower bound is a consequence of (6.2) and the fact that $u\left(t, \alpha^{*}\right)=\frac{1}{C_{1} \cdot(T-t)}$. For the upper bound we discuss two cases. If $\alpha$ is such that $u_{0}(\alpha) \leq 0$, then by (6.2) we obtain $|u(t, \alpha)| \leq\left\|u_{0}\right\|_{L_{x}^{\infty}\left(\mathbb{R}^{2}\right)}$. If $\alpha$ is such that $u_{0}(\alpha)>0$, then again by (6.2), we have

$$
|u(t, \alpha)| \leq \frac{1}{C_{1}} \cdot \frac{1}{\frac{1}{C_{1} u_{0}(\alpha)}-t} \leq \frac{1}{C_{1}} \cdot \frac{1}{T-t},
$$

where the last inequality follows from the fact that $u_{0}(\alpha) \leq u_{0}\left(\alpha^{*}\right)=\frac{1}{C_{1} T}$. The theorem is proved.

\section{Proof of Theorem 1.10}

To prove the formation of finite time singularities, we introduce the following set of functions.

Definition 7.1. (Admissible initial conditions). Let $0<\delta<1, a>0, b>0$ be constants, the set $A_{\delta, a, b}$ consists of nonnegative functions $f \in L_{x}^{1}((1+$ $\left.|x|^{2}\right) d x$ ) satisfying the following conditions:

1. The $L_{x}^{1}$ mass of $f$ is localized near the origin:

$$
\int_{|x| \geq \frac{\delta}{2}} f(x) d x \leq \frac{\delta}{4}\|f\|_{L_{x}^{1}}
$$


2. $f$ satisfies the following inequality:

$$
\int_{\mathbb{R}^{2}} f(x)|x|^{2} d x-a\|f\|_{L_{x}^{1}}+b<0 .
$$

Remark 7.2. The first condition in the above definition quantifies the fact that the initial mass of the solution is concentrated near the origin. The second condition is a technical condition which we need to prove by contradiction the existence of finite time singularities (see (7.9)).

Lemma 7.3. For any $0<\delta<1, a>0, b>0$, the set $A_{\delta, a, b}$ defined in 7.1 is nonempty. Moreover, $A_{\delta, a, b} \cap L^{1} \cap H^{s}$ is nonempty.

Proof. This is almost trivial. Let $0<\delta<1, a>0, b>0$ be given. Take a function $\psi \in C_{c}^{\infty}\left(\mathbb{R}^{2}\right), \psi \geq 0$ such that

$$
-a \frac{\|\psi\|_{L_{x}^{1}}^{2}}{\|\psi\|_{L_{x}^{1}}+1}+b<0
$$

Then consider the rescaled function $\psi_{\epsilon}(x):=\epsilon^{-2} \psi(x / \epsilon)$. This scaling transformation leaves the $L_{x}^{1}$ norm unchanged. Then consider $f=\psi_{\epsilon}$. For sufficiently small $\epsilon>0$, it is not difficult to see that the first inequality in Definition 7.1 is satisfied for $f=\psi_{\epsilon}$. For the second inequality, just note that

$$
\int_{\mathbb{R}^{2}} \psi_{\epsilon}|x|^{2} d x=\epsilon^{4} \int_{\mathbb{R}^{2}} \psi(x)|x|^{2} d x \rightarrow 0
$$

as we take $\epsilon \rightarrow 0$. This together with (7.1) and the fact that $\left\|\psi_{\epsilon}\right\|_{L_{x}^{1}}=\|\psi\|_{L_{x}^{1}}$ proves the second inequality in Definition 7.1 for $\psi_{\epsilon}$. The lemma is proved.

We are going to take $\phi \in C_{c}^{\infty}\left(\mathbb{R}^{2}\right)$ with $0 \leq \phi \leq 1$, and

$$
\phi(x)= \begin{cases}1, & |x| \leq 1 \\ 0, & |x| \geq 2\end{cases}
$$

Lemma 7.4. (Mass localization for short time). Let $u_{0} \in L_{x}^{1}\left(\mathbb{R}^{2}\right) \cap H_{x}^{s}\left(\mathbb{R}^{2}\right)$ for some $s>3$. Assume $u_{0} \in A_{\delta, a, b}$ for some $a>0, b>0,0<\delta<\frac{1}{4}$. Let $u$ be the corresponding solution and assume it is global. Then there exists a constant $C=C(\delta, \alpha)>0$ and

$$
T=\frac{\delta}{C \cdot\left(\left\|u_{0}\right\|_{L_{x}^{1}\left(\mathbb{R}^{2}\right)}+\nu\right)}
$$

such that

$$
\sup _{0 \leq t \leq T} \int_{|x| \geq \delta} u(t, x) d x \leq \delta\left\|u_{0}\right\|_{L_{x}^{1}}
$$


Proof . We take $\phi_{\delta}(x):=\phi(2 x / \delta)$ where $\phi$ is defined in (7.2). Then since the $L_{x}^{1}$ norm of the solution is preserved, we have by using (1.2),

$$
\begin{aligned}
& \frac{d}{d t} \int_{\mathbb{R}^{2}} u(t, x)\left(1-\phi_{\delta}(x)\right) d x=-\frac{d}{d t} \int u(t, x) \phi_{\delta}(x) d x \\
& =\int \nabla \cdot(u B(u)) \phi_{\delta}(x) d x+\nu \int(-\Delta)^{\alpha / 2} u \phi_{\delta} d x \\
& =\int_{\mathbb{R}^{2}} \int_{\mathbb{R}^{2}} u(t, x) u(t, y) \frac{x-y}{|x-y|^{2}} \cdot \nabla \phi_{\delta}(x) d x d y+\nu \int_{\mathbb{R}^{2}} u(t, x)(-\Delta)^{\alpha / 2} \phi_{\delta} d x .
\end{aligned}
$$

Symmetrizing the last double integral in $x$ and $y$ gives us

$$
\begin{aligned}
\frac{d}{d t} \int_{\mathbb{R}^{2}} u(t, x)( & \left.-\phi_{\delta}(x)\right) d x \\
= & \frac{1}{2} \int_{\mathbb{R}^{2}} \int_{\mathbb{R}^{2}} u(t, x) u(t, y) \frac{(x-y) \cdot\left(\nabla \phi_{\delta}(x)-\nabla \phi_{\delta}(y)\right)}{|x-y|^{2}} d x d y \\
& +\nu \int_{\mathbb{R}^{2}} u(t, x)(-\Delta)^{\alpha / 2} \phi_{\delta}(x) d x \\
\leq & \left\|u_{0}\right\|_{L_{x}^{1}}^{2} \cdot C_{1}\left\|\partial_{i} \partial_{j} \phi_{\delta}\right\|_{L_{x}^{\infty}}+\nu\left\|u_{0}\right\|_{L_{x}^{1}}\left\|(-\Delta)^{\alpha / 2} \phi_{\delta}\right\|_{L_{x}^{\infty}},
\end{aligned}
$$

where $C_{1}$ is a positive constant. The quantity $\left\|(-\Delta)^{\alpha / 2} \phi_{\delta}\right\|_{L_{x}^{\infty}}$ can be easily bounded through its Fourier transform and therefore is finite due to our assumption on $\phi$ :

$$
\left\|(-\Delta)^{\alpha / 2} \phi_{\delta}\right\|_{L_{x}^{\infty}} \leq\left\||\xi|^{\alpha} \hat{\phi}_{\delta}(\xi)\right\|_{L_{\xi}^{1}}<\infty .
$$

Putting together the estimates (7.3) and (7.4), we obtain

$$
\frac{d}{d t} \int_{\mathbb{R}^{2}} u(t, x)\left(1-\phi_{\delta}(x)\right) d x \leq C_{2} \cdot\left(\left\|u_{0}\right\|_{L_{x}^{1}}^{2}+\nu\left\|u_{0}\right\|_{L_{x}^{1}}\right),
$$

where $C_{2}=C_{2}\left(\left\|\partial_{i} \partial_{j} \phi_{\delta}\right\|_{L_{x}^{\infty}},\left\|(-\Delta)^{\alpha / 2} \phi_{\delta}\right\|_{L_{x}^{\infty}}\right)$ is a positive constant. Now it is not difficult to see that if we take

$$
T=\frac{\delta\left\|u_{0}\right\|_{L_{x}^{1}}}{2 C_{2} \cdot\left(\left\|u_{0}\right\|_{L_{x}^{1}}^{2}+\nu\left\|u_{0}\right\|_{L_{x}^{1}}\right)},
$$

then for any $0 \leq t \leq T$, by (7.5) we have

$$
\begin{aligned}
\int_{|x| \geq \delta} u(t, x) d x & \leq \int_{\mathbb{R}^{2}} u(t, x)\left(1-\phi_{\delta}(x)\right) d x \leq \int_{\mathbb{R}^{2}} u_{0}(x)\left(1-\phi_{\delta}(x)\right) d x+\frac{\delta}{2}\left\|u_{0}\right\|_{L_{x}^{1}} \\
& \leq \int_{|x| \geq \frac{\delta}{2}} u_{0}(x) d x+\frac{\delta}{2}\left\|u_{0}\right\|_{L_{x}^{1}} \leq \delta\left\|u_{0}\right\|_{L_{x}^{1}},
\end{aligned}
$$

where the last inequality follows from our assumption on $u_{0}$. The lemma is proved. 
Now we are ready to prove Theorem 1.10.

Proof of Theorem 1.10. We argue by contradiction. Assume the initial data $u_{0} \in H_{x}^{s}\left(\mathbb{R}^{2}\right) \cap L_{x}^{1}\left(\mathbb{R}^{2}\right)$ for some $s>3$ and $u_{0}$ is nonnegative with nonzero $L_{x}^{1}$ mass. Assume $u_{0} \in A_{\delta, a, b}$ for some $0<\delta<\frac{1}{4}, a>0, b>0$ whose values are to specified in the course of the proof. Let $w(x)=|x|^{2} \phi(x)$ where $\phi$ is defined in (7.2). We first compute the truncated virial integral

$$
\begin{aligned}
& \frac{d}{d t} \int_{\mathbb{R}^{2}} u(t, x) w(x) d x=\int_{\mathbb{R}^{2}} u B(u) \cdot \nabla w d x-\nu \int_{\mathbb{R}^{2}} u(-\Delta)^{\alpha / 2} w d x \\
& =-\int_{\mathbb{R}^{2}} \int_{\mathbb{R}^{2}} u(t, x) u(t, y) \frac{(x-y) \cdot \nabla w(x)}{|x-y|^{2}} d x d y-\nu \int_{\mathbb{R}^{2}} u(-\Delta)^{\alpha / 2} w d x
\end{aligned}
$$

$$
=-\frac{1}{2} \int_{\mathbb{R}^{2}} \int_{\mathbb{R}^{2}} u(t, x) u(t, y) \frac{(x-y) \cdot(\nabla w(x)-\nabla w(y))}{|x-y|^{2}} d x d y-\nu \int_{\mathbb{R}^{2}} u(-\Delta)^{\alpha / 2} w d x,
$$

where the last equality follows from symmetrizing the integral in $x$ and $y$. We now break the last double integral into two parts. Denote

$$
\Omega_{1}=\left\{(x, y) \in \mathbb{R}^{2} \times \mathbb{R}^{2}:|x| \leq \frac{1}{4},|y| \leq \frac{1}{4}\right\} .
$$

On $\Omega_{1}$ we have $w(x)=|x|^{2}$ and $w(y)=|y|^{2}$ due to our assumption on $\phi$. Therefore

$$
\begin{aligned}
\int_{\Omega_{1}} u(t, x) u(t, y) & \frac{(x-y) \cdot(\nabla w(x)-\nabla w(y))}{|x-y|^{2}} d x d y \\
& =2 \int_{\Omega_{1}} u(t, x) u(t, y) d x d y=2\left(\int_{|x| \leq \frac{1}{4}} u(t, x) d x\right)^{2} .
\end{aligned}
$$

For those pairs $(x, y) \notin \Omega_{1}$, we have

$$
|\nabla w(x)-\nabla w(y)| \leq C_{1}\left\|\partial_{i} \partial_{j} w\right\|_{L_{x}^{\infty}}|x-y|,
$$

where $C_{1}$ is an absolute positive constant. Therefore we have the estimate

$$
\begin{aligned}
\mid \int_{\mathbb{R}^{2} \times \mathbb{R}^{2} \backslash \Omega_{1}} u(t, x) u(t, y) & \frac{(x-y) \cdot(\nabla w(x)-\nabla w(y))}{|x-y|^{2}} d x d y \mid \\
& \leq C_{1}\left\|\partial_{i} \partial_{j} w\right\|_{L_{x}^{\infty}} \int_{\mathbb{R}^{2} \times \mathbb{R}^{2} \backslash \Omega_{1}} u(t, x) u(t, y) d x d y \\
& \leq 2 C_{1}\left\|\partial_{i} \partial_{j} w\right\|_{L_{x}^{\infty}}\left\|u_{0}\right\|_{L_{x}^{1}} \int_{|x| \geq \frac{1}{4}} u(t, x) d x .
\end{aligned}
$$


Collecting the estimates (7.6), (7.7) and (7.8), we have

$$
\begin{aligned}
& \frac{d}{d t} \int_{\mathbb{R}^{2}} u(t, x) w(x) d x \leq-\left(\int_{|x| \leq \frac{1}{4}} u(t, x) d x\right)^{2} \\
& \quad+C_{1}\left\|\partial_{i} \partial_{j} w\right\|_{L_{x}^{\infty}}\left\|u_{0}\right\|_{L_{x}^{1}} \int_{|x| \geq \frac{1}{4}} u(t, x) d x+\nu\left\|u_{0}\right\|_{L_{x}^{1}}\left\|(-\Delta)^{\alpha / 2} w\right\|_{L_{x}^{\infty}} .
\end{aligned}
$$

By Lemma 7.4 and the choice of $T$, we then obtain for each $0 \leq t \leq T$,

$$
\begin{aligned}
& \frac{d}{d t} \int_{\mathbb{R}^{2}} u(t, x) w(x) d x \leq \\
& \quad \leq\left(-(1-\delta)^{2}+C_{1}\left\|\partial_{i} \partial_{j} w\right\|_{L_{x}^{\infty}} \delta\right)\left\|u_{0}\right\|_{L_{x}^{1}}^{2}+\nu\left\|(-\Delta)^{\alpha / 2} w\right\|_{L_{x}^{\infty}}\left\|u_{0}\right\|_{L_{x}^{1}} .
\end{aligned}
$$

Integrating over $[0, T]$ gives us

$$
\begin{aligned}
\int_{\mathbb{R}^{2}} u(T, x) w(x) d x \leq \\
\leq \int_{\mathbb{R}^{2}} u_{0}(x) w(x) d x+\frac{\delta\left(-(1-\delta)^{2}+C_{1}\left\|\partial_{i} \partial_{j} w\right\|_{L_{x}^{\infty}} \delta\right)\left\|u_{0}\right\|_{L_{x}^{1}}^{2}}{C \cdot\left(\left\|u_{0}\right\|_{L_{x}^{1}}+\nu\right)} \\
\quad+\frac{\delta\left\|(-\Delta)^{\alpha / 2} w\right\|_{L_{x}^{\infty}}\left\|u_{0}\right\|_{L_{x}^{1}}}{C \cdot\left(\left\|u_{0}\right\|_{L_{x}^{1}}+\nu\right)},
\end{aligned}
$$

where the constant $C$ is the same as in Lemma 7.4. Now let $\delta$ be sufficiently small such that

$$
-(1-\delta)^{2}+C_{1}\left\|\partial_{i} \partial_{j} w\right\|_{L_{x}^{\infty}} \delta \leq-\frac{1}{2}
$$

We can choose such a $\delta$ because $C_{1}$ is an absolute constant and $w$ is a fixed function. With this choice of $\delta$, we have

$$
\begin{aligned}
\int_{\mathbb{R}^{2}} u(T, x) & w(x) d x \leq \\
& \leq \int_{\mathbb{R}^{2}} u_{0}(x) w(x) d x-\frac{\delta}{2 C}\left\|u_{0}\right\|_{L_{x}^{1}}+\frac{\delta\left\|(-\Delta)^{\frac{\alpha}{2}} w\right\|_{L_{x}^{\infty}}}{C} \\
& \leq \int_{\mathbb{R}^{2}} u_{0}(x)|x|^{2} d x-\frac{\delta}{2 C}\left\|u_{0}\right\|_{L_{x}^{1}}+\frac{\delta\left\|(-\Delta)^{\frac{\alpha}{2}} w\right\|_{L_{x}^{\infty}}}{C} \\
& <0
\end{aligned}
$$

where the last inequality holds as long as we choose our initial data $u_{0} \in$ $A_{\delta, a, b}$ with $a=\frac{\delta}{2 C}$ and $b=\frac{\delta\left\|(-\Delta)^{\frac{\alpha}{2}} w\right\|_{L_{x}^{\infty}}}{C}$. But this is a contradiction since

$$
\int_{\mathbb{R}^{2}} u(T, x) w(x) d x \geq 0 .
$$

Finally it is easy to see that the set of values $(\delta, a, b)$ is open for which our previous argument goes through. The theorem is proved. 


\section{References}

[1] Bardos, C., Penel, P., Frisch, U. and Sulem, P. L.: Modified dissipativity for a nonlinear evolution equation arising in turbulence. Arch. Rational Mech. Anal. 71 (1979), no. 3, 237-256.

[2] Biler, P., Hebisch, W. and Nadzieja, T.: The Debye system: existence and large time behavior of solutions. Nonlinear Anal. 23 (1994), no. 9, 1189-1209.

[3] Biler, P.: Existence and nonexistence of solutions for a model of gravitational interaction of particles, III. Colloq. Math. 68 (1995), no. 2, 229-239.

[4] Biler, P.: The Cauchy problem and self-similar solutions for a nonlinear parabolic equation. Studia Math. 114 (1995), no. 2, 181-205.

[5] Biler, P.: Local and global solutions of a nonlinear nonlocal parabolic problem. In Nonlinear analysis and applications, 49-66. Gakuto Int. ser. Math. Sci. and Its Appl. 7. Gakkōtosho, Tokyo, 1996.

[6] Biler, P. And Nadzieja, T.: A class of nonlocal parabolic problems occurring in statistical mechanics. Colloq. Math. 66 (1993), no. 1, 131-145.

[7] Biler, P. And Nadzieja, T.: Existence and nonexistence of solutions for a model of gravitational interaction of particles I. Colloq. Math. 66 (1994), no. 2, 319-334.

[8] Biler, P. And WoyczyŃski, W. A.: Global and exploding solutions for nonlocal quadratic evolution problems. SIAM J. Appl. Math. 59 (1999), no. $3,845-869$.

[9] Brenner, M. P., Constantin, P., Kadanoff, L. P., Schenkel, A. and Venkataramani, S. C.: Diffusion, attraction and collapse. Nonlinearity 12 (1999), no. 4, 1071-1098.

[10] Constantin, P., Majda A. And Tabak, E.: Formation of strong fronts in the 2D quasi-geostrophic thermal active scalar. Nonlinearity 7 (1994), no. $6,1495-1533$.

[11] Córdoba, A. And Córdoba, D. A maximum principle applied to quasigeostrophic equations. Comm. Math. Phys. 249 (2004), no. 3, 511-528.

[12] Dawson, D. A. And Gorostiza, L. G.: Generalized solutions of a class of nuclear-space-valued stochastic evolution equations. Appl. Math. Optim. 22 (1990), no. 3, 241-263.

[13] Ju, N.: The maximum principle and the global attractor for the dissipative 2D quasi-geostrophic equations. Comm. Math. Phys. 255 (2005), 161-181.

[14] Keller, E. F. And Segel L. A.: Initiation of slime mold aggregation viewed as an instability. J. Theor. Biol. 26 (1970), 399-415.

[15] Komatsu, T.: Markov processes associated with certain integro-differential operators. Osaka J. Math. 10 (1973), 271-303.

[16] Komatsu, T.: On the martingale problem for generators of stable processes with perturbations. Osaka J. Math. 21 (1984), no. 1, 113-132. 
[17] Li, D. And Rodrigo, J.: Finite-time singularities of an aggregation equation in $\mathbb{R}^{n}$ with fractional dissipation. Comm. Math. Phys. 287 (2009), no. $2,687-703$.

[18] Li, D. And Rodrigo, J.: Blow up of solutions for a 1D transport equation with nonlocal velocity and supercritical dissipation. Adv. Math. 217 (2008), no. 6, 2563-2568.

[19] Li, D. And Rodrigo, J.: Blow up for the generalized surface quasigeostrophic equation with supercritical dissipation. Comm. Math. Phys. 286 (2009), no. 1, 111-124.

[20] Majda, A. J. And Bertozzi, A. L.: Vorticity and incompressible flow. Texts in Applied Math. 27. Cambridge Univ. Press, Cambridge, UK, 2002.

[21] Stroock, D. W.: Diffusion processes associated with Lévy generators. Z. Wahrscheinlichkeitstheorie und Verw. Gebiete 32 (1975), no. 3, 209-244.

[22] TaO, T., Visan, M. And Zhang, X.: Global well-posedness and scattering for the defocusing mass-critical nonlinear Schrodinger equation for radial data in high dimensions. Duke Math. J. 140 (2007), no. 1, 165-202.

[23] Wolansky, G. On steady distributions of self-attracting clusters under friction and fluctuations. Arch. Rational Mech. Anal. 119 (1992), 355-391.

Recibido: 3 de septiembre de 2008

Dong Li

Department of Mathematics

University of Iowa

14 MacLean Hall, Iowa City, Iowa 52240, USA

Dong-Li@uiowa.edu

José L. Rodrigo

Warwick University

Zeeman Building

Coventry, CV47AL, UK

j.rodrigo@warwick.ac.uk

Xiaoyi Zhang

Department of Mathematics

University of Iowa

14 MacLean Hall, Iowa City, Iowa 52240, USA

xiaoyi-zhang@uiowa.edu

D. Li and X. Zhang were partially supported by the National Science Foundation under agreement no. DMS-0635607. D. Li was partially supported by a start-up funding from the Math. Department of University of Iowa and the NSF no. DMS-0908032. X. Zhang was also supported by NSF grant no. 10601060 and project 973 in China. J. Rodrigo was partially supported by MTM2005-05980, Ministerio de Educación y Ciencia (Spain). 